\title{
PFEM-DEM for particle-laden flows with free surface
}

\author{
Alessandro Franci ${ }^{*} \dagger$ - Ignasi \\ de-Pouplana* $^{\dagger}$. Guillermo Casas ${ }^{*} \dagger$. \\ Miguel Ángel Celigueta ${ }^{*, \dagger}$. Joaquín

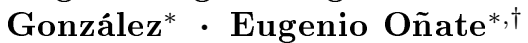

Received: date / Accepted: date

\begin{abstract}
This work proposes a fully Lagrangian formulation for the numerical modeling of free-surface particle-laden flows. The fluid phase is solved using the Particle Finite Element Method (PFEM), while the solid particles embedded in the fluid are modeled with the Discrete Element Method (DEM). The coupling between the implicit PFEM and the explicit DEM is performed through a sub-stepping staggered scheme. This work only considers suspended spherical particles that are assumed not to affect the fluid motion. Several tests are presented to validate the formulation. The PFEM-DEM results show very good agreement with analytical solutions, laboratory tests and numerical results from alternative numerical methods.
\end{abstract}

Keywords PFEM · DEM - CFD-DEM · particulate flow · particle-laden flow $\cdot$ free-surface

\footnotetext{
* Centre Internacional de Mètodes Numèrics a l'Enginyeria (CIMNE)

Edifici C1, Campus Nord UPC, Carrer del Gran Capità, S/N, 08034 Barcelona

Tel.: +34934017495

Fax: +34934016517

$\dagger$ Universitat Politecnica de Catalunya (UPC)

Campus Nord, Carrer de Jordi Girona, 1, 08034 Barcelona

Tel.: +34934016200

Fax: +34934016517

E-mail: falessandro@cimne.upc.edu
} 


\section{Introduction}

The term Particulate, or particle-laden flow refers to the dynamics of a multicomponent medium [36] where at least one component or phase is formed by a continuously connected fluid (the suspending fluid), and where at least one of the remaining phases is formed by a number of disconnected sub-domains (the particles) which are surrounded by the fluid. Typically, the particles are assumed to be immiscible, numerous and small with respect to the characteristic size of the domain considered. In this work, we restrict our attention to bi-component flows involving suspensions of small, rigid-solid spheres in an incompressible Newtonian fluid.

During the last decade or so, there has been a growing interest in the development of numerical techniques for the simulation of particle-laden flows, perhaps due to the ever-increasing availability of computational resources. Indeed, simulating such systems often requires the consideration of a wide range of scales simultaneously $[43,44]$, as it is difficult to model the intricate details of the particle-scale phenomena that are needed for a fully macroscopic description.

There exist roughly three main families (one may also consider a fourth one, see [45]) of numerical methods for particle-laden flows, each corresponding to a different combination of scales at which each phase is described, see, e.g. [34]:

1. Fully Resolved Approach (FRA): Here the details of the flow around each particle are numerically resolved and the whole system reduces to a fluidstructure interaction problem, possibly including inter-particle contact as well. The motion of the particles, normally taken as rigid solids, can be calculated with a variety of methods, such as the Discrete Element Method (DEM) [45]. The FRA can be understood as a 'brute-force' solution; reliable, but only practical for very small systems.

2. Multi-Fluid Approach (MFA): In this approach, the medium is described as a multicomponent continuum and the solving equations are obtained by applying an average or filter $[46,37]$ to the differential equations that describe each phase separately. Unfortunately, the resulting averaged system contains terms that cannot be expressed in terms of the filtered fields, so closure models have to be introduced in order to obtain a well-posed problem $[35,36]$. The closed system of equations can be discretized and solved with a variety of Computational Fluid Dynamics (CFD) solvers, e.g. based on the Finite Difference Method (FDM), the Finite Volume Method (FVM) or the Finite Element Method (FEM).

3. Hybrid approach: In this approach, the particles are individually tracked but the details of the flow around them are smoothed. This is achieved by using the equations that correspond to the fluid phase, as in the MFA, but replacing the ones for the particles phase by the equations of motion of the individual particles, as in the FRA. To couple the fluid and the particles, the smoothed fluid fields are evaluated at the location of the particles' centers. The motion of the particles can be derived using closure models, such as an empirical drag law, to achieve forward coupling to the 
fluid phase. The particle-to-fluid coupling (backward coupling) can, in turn, be achieved by repeatedly applying a discrete filtering operation on the particles before solving for the fluid phase.

In this work, we choose to focus on the hybrid approach. For the description of the particles, we use the discrete element method (DEM), while, the Particle Finite Element Method (PFEM) [1] is used as the CFD solver. The DEM has been chosen for modeling the solid phase of the particle-laden flow, as this method is robust in treating collisions and sustained contact both between particles and between particles and solid boundaries. On the other hand, the PFEM, basing on a Lagrangian description of the fluid motion, allows for a natural tracking of fluid interfaces and it can be safely used for large deformation processes, such as the ones occurring in unsteady free-surface fluid flows $[2,3]$, fluid-structure interaction problems [5,10], granular flows [8,9], bed erosion [6] and geotechnical applications [7]. Such choices define our method as a PFEM-DEM approach, hence a subcategory of the so-called CFD-DEM methods $[67,52,53,55,54,61,62]$. The CFD-DEM has been used directly in industrial problems such as fluid catalytic cracker (FCC) units [49], pneumatic transport [50] and mud-flow transport problems from the oil industry [51]. But perhaps the most promising use of CFD-DEM procedures is in the derivation of empirical relations and validation examples $[47,48]$, which can be used to improve the models used in coarser but more affordable techniques.

Here we will concentrate on a one-way-only coupled strategy, where we neglect the effect of the particles on the flow. Such strategy is suitable for sufficiently disperse flows [66] (see also the second chapter in [37]), which arise in many natural and industrial problems like contaminant dispersion, the study of sprays and the manufacture of composites. Under such hypothesis, the formulation becomes simpler and it brings about certain advantages. For example, it makes it possible to calculate the fluid first, store the results, and then reuse them multiple times with different particle ensembles. Nonetheless, our formulation can indeed be extended to the fully two-way coupled case, which is left for future work.

In contrast to previous PFEM-DEM formulations [39,40], where the PFEM mesh nodes were assumed to be coincident with the suspended spherical elements, in this work the solid particles are free to move across the fluid elements of the PFEM mesh, such as in the traditional particle-in-cell scheme $[57,56]$. This is extremely helpful in order to maintain a good quality of the mesh during the analysis at a reasonable cost, the reason being that the solid particles do not impose any restriction to the PFEM remeshing procedure.

Our aim is to present the details of the PFEM-DEM approach, provide the basic instructions on how to implement it and discuss some of its particularities. This work should pave the way for other researchers interested in taking advantage of the PFEM features for the analysis of particle-laden flow problems: robust performance with free surfaces and intersecting interfaces and versatile FSI capabilities, and easiness of coupling with the DEM. 
The paper is organized as follows. In Section 2 we introduce the continuum model for the fluid phase and the equation of motion for the particles. In Section 3 we describe the proposed PFEM-DEM algorithm, including a brief account of the fluid-phase solution, the particle-phase solution strategies and the coupling strategy. In Section 6 we present four benchmark examples to validate the proposed PFEM-DEM approach. The goal is to show its applicability to problems of industrial interest and to illustrate different aspects of the method, such as the effect of the PFEM remeshing on the DEM solution and the possibility of using the DEM elements as tracers of the PFEM solution.

\section{Governing equations}

This section presents the governing equations for the particle-laden flow problem. In the approach taken in this work, the fluid is assumed to be insensitive to the solid particles motion. On the contrary, the particles are affected by the fluid solution. Considering these hypotheses, the governing equations for the fluid phase and the solid particles are those presented in Section 2.1 and Section 2.2, respectively.

\subsection{Fluid framework}

The fluid is governed by the Navier-Stokes equations, i.e. the balance of linear momentum and the mass conservation equations.

Considering an Updated Lagrangian framework as in the standard PFEM [1], the linear momentum equations read

$$
\rho_{f} \frac{\partial \boldsymbol{u}}{\partial t}-\nabla \cdot \boldsymbol{\sigma}-\rho_{f} \boldsymbol{g}=\mathbf{0} \quad \text { in } \quad \Omega_{f} \times(0, T)
$$

where $\rho_{f}$ is the fluid density, $\boldsymbol{u}$ is the fluid velocity vector, $t$ is the time, $\boldsymbol{\sigma}$ is the Cauchy stress tensor, $\boldsymbol{g}$ is the gravity acceleration vector, $\Omega_{f}$ is the updated/deformed fluid domain and $(0, T)$ time interval considered.

A standard Newtonian law is considered for the fluid phase. Thus, the Cauchy stress tensor is computed as

$$
\boldsymbol{\sigma}=p \boldsymbol{I}+2 \mu_{f} \boldsymbol{d}^{\prime}
$$

where $p$ is the pressure, $\boldsymbol{I}$ is the $2^{\text {nd }}$ order identity tensor, $\mu_{f}$ is the fluid dynamic viscosity and $\boldsymbol{d}^{\prime}$ is the deviatoric part of the deformation rate tensor $\boldsymbol{d}$, defined as

$$
\boldsymbol{d}^{\prime}=\frac{1}{2}\left(\nabla \boldsymbol{u}+(\nabla \boldsymbol{u})^{T}\right)-\frac{1}{3} d_{v} \boldsymbol{I}
$$

where, in $3 \mathrm{D}, d_{v}=\frac{\partial u_{x}}{\partial x}+\frac{\partial u_{y}}{\partial y}+\frac{\partial u_{z}}{\partial z}$. 
Following [11-13] a small compressibility is allowed for the fluid medium. Hence, the mass conservation equation is written as

$$
\nabla \cdot \boldsymbol{u}-\frac{1}{\kappa_{f}} \frac{\partial p}{\partial t}=0 \quad \text { in } \quad \Omega_{f} \times(0, T)
$$

where $\kappa_{f}$ is the bulk modulus of the fluid.

Remark 1 For the fluid bulk modulus going to infinity the standard divergencefree form of the continuity equation is recovered $(\nabla \cdot \boldsymbol{u}=0)$.

The fluid governing equations set is closed by the following boundary conditions

$$
\begin{array}{ll}
\boldsymbol{u}-\hat{\boldsymbol{u}}=\mathbf{0} & \text { on } \Gamma_{f}^{v} \\
\boldsymbol{\sigma} \cdot \boldsymbol{n}-\hat{\boldsymbol{t}}=\mathbf{0} & \text { on } \Gamma_{f}^{t}
\end{array}
$$

where $\boldsymbol{n}$ is the normal vector to fluid boundaries, and $\hat{\boldsymbol{u}}$ and $\hat{\boldsymbol{t}}$ are the velocities and tractions prescribed at Dirichlet $\left(\Gamma_{f}^{v}\right)$ and Neumann $\left(\Gamma_{f}^{t}\right)$ boundaries, respectively.

\subsection{Particles framework}

The equations of motion for a given spherical particle read

$$
\begin{aligned}
m_{p} \frac{\mathrm{d} \boldsymbol{v}}{\mathrm{d} t} & =\mathbf{F}_{\mathrm{B}}+\mathbf{F}_{\text {hydro }}+\sum_{k_{p n}} \mathbf{F}_{k_{p n}}+\sum_{k_{w n}} \mathbf{F}_{k_{w n}} \\
I_{p} \frac{\mathrm{d} \boldsymbol{\omega}_{p}}{\mathrm{~d} t} & =\boldsymbol{T}_{\text {hydro }}+\boldsymbol{T}_{\text {contact }}
\end{aligned}
$$

where $m_{p}$ is the mass of the particle, $\boldsymbol{v}$ the velocity of the particle's center, $\boldsymbol{F}_{\mathrm{B}}$ the Buoyancy force, $\boldsymbol{F}_{\text {hydro }}$ the hydrodynamic force, and $\mathbf{F}_{k_{p n}}$ and $\mathbf{F}_{k_{w n}}$ the interaction forces among the particles and between the particles and the rigid walls, respectively. In the second equation $I_{p}$ is the moment of inertia, $\boldsymbol{\omega}_{p}$ the angular velocity, and $\boldsymbol{T}_{\text {hydro }}$ and $\boldsymbol{T}_{\text {contact }}$ the hydrodynamic torque and the torque produced by the contact forces. The indices $k_{p n}$ and $k_{w n}$ run over all the neighboring particles and triangular rigid boundaries, respectively (Section 4).

The Buoyancy force $\boldsymbol{F}_{\mathrm{B}}$ is defined as

$$
\boldsymbol{F}_{\mathrm{B}}=\left(m_{p}-m_{f}\right) \boldsymbol{g}
$$

where $m_{f}$ is the mass of the volume of fluid displaced by the particle.

The hydrodynamic force term $\boldsymbol{F}_{\text {hydro }}$ is the result of the relative motion of the particle with respect to the background fluid. It can be modeled by the following split [32], valid in a wide range of regimes,

$$
\boldsymbol{F}_{\text {hydro }}=\boldsymbol{F}_{\mathrm{U}}+\boldsymbol{F}_{\mathrm{A}}+\boldsymbol{F}_{\mathrm{D}}
$$

Let us briefly discuss the particular expressions of the different terms in Eq.(8). 
$\boldsymbol{F}_{\mathrm{U}}$ represents the force that the sphere of fluid displaced by the particle would feel if taken as a point-mass and it is computed as

$$
\boldsymbol{F}_{\mathrm{U}}=m_{f} \frac{\mathrm{D} \boldsymbol{u}}{\mathrm{D} t}
$$

where the capitalized derivative operator denotes the material derivative. Note that in a Lagrangian description of the continuum there is no distinction between material and partial time derivatives, so that $\frac{\mathrm{D}}{\mathrm{D} t} \equiv \frac{\partial}{\partial t}$.

Remark 2 As argued in [32], the expression of $\boldsymbol{F}_{\mathrm{U}}$ holds for both the very low and very high particle Reynolds numbers, as defined below; see Eq.(12). This confirms that the expression should be robust generically.

The added mass force $\boldsymbol{F}_{\mathrm{A}}$ results from the resistance of the fluid to the motion induced by the particle. The following expression holds both in the inviscid and the vanishing particle Reynolds number limits,

$$
\boldsymbol{F}_{\mathrm{A}}=\frac{1}{2} m_{f}\left(\frac{\mathrm{D} \boldsymbol{u}}{\mathrm{D} t}-\frac{\mathrm{d} \boldsymbol{v}}{\mathrm{d} t}\right)
$$

Note that in the equation above $\frac{\mathrm{D}}{\mathrm{d} t}$ represents material derivation of the fluid field, or derivation following the fluid, while $\frac{\mathrm{d}}{\mathrm{d} t}$ represents derivation following the particle.

There is considerable evidence of the accuracy of Eq.(10) outside its theoretical range of validity $[58,59]$. We thus assume this form of the added mass force to hold for the full range of Reynolds numbers too.

Finally, the drag force $\boldsymbol{F}_{\mathrm{D}}$ can be defined as the ensemble-averaged force experimented by a particle submerged in a statistically stationary flow, in the direction of the relative velocity between the particle and the far-field averaged flow velocity. It can be expressed as

$$
\boldsymbol{F}_{D}=\frac{1}{2} \rho_{p} A_{p} C_{D}\|\boldsymbol{w}\|(\boldsymbol{w})
$$

where $\rho_{p}$ is the particle's density, $A_{p}$ is the cross-sectional area of the particle for a section orthogonal to $\boldsymbol{w}:=\boldsymbol{u}-\boldsymbol{v}$, and $C_{D}$ is the drag coefficient.

The drag coefficient is in general dependent on the particle-based Reynolds number $R e_{p}$, the shape of the particle [33], the local solid fraction [60] and the properties of the fluid. This formulation is a generalization of the Stokes drag, for which the drag coefficient is $C_{D, \text { Stokes }}=24 / R e_{p}$, and

$$
R e_{p}:=\frac{d_{p}|\boldsymbol{w}|}{\nu_{f}}
$$

where $d_{p}$ is the particle's diameter and $\nu_{f}$ the kinematic viscosity of the fluid.

In the examples presented in this work we use the empirical generalization by [63], defined by

$$
C_{D}\left(R e_{p}\right)=\left\{\begin{array}{cc}
\left(1+0.15 R e_{p}^{0.687}\right) C_{D, \text { Stokes }} & \text { if } R e_{p} \leq 1000 \\
0.44 & \text { otherwise }
\end{array}\right.
$$


which, for our purposes, is sufficiently accurate for particle Reynolds numbers up to well over 1000 .

Remark 3 In this work, we neglect both the history and the lift forces. The former represents the unsteady part of the viscous resistance (where $\boldsymbol{F}_{\mathrm{D}}$ is its steady part) to relative velocities of the particle with respect to the background fluid. The lift can be defined as the component of the hydrodynamic force orthogonal to $\boldsymbol{w}$. In the examples presented in the paper, the effect of these terms is considered as negligible versus the other forces in Eq.(6). An accurate study of the history and lift forces in CFD-DEM analyses can be found in [37].

\section{Fluid solution scheme}

In this work, the fluid phase is solved with the PFEM using an implicit twostep velocity-pressure scheme, proposed and validated in [17]. The governing equations are discretized as in the standard Galerkin FEM fashion. Linear shape functions are used for the approximation of both the velocity and the pressure fields. It is well known that this combination does not fulfill the $L B B$ condition [16]. In this work, the required stabilization is introduced via the Finite Increment Calculus (FIC) technique $[18,17,19]$. The derivation of the FIC-FEM form of the governing equations has been detailed in [17], and here only the final fully-discretized and linearized form is given.

The fluid solution is obtained by solving iteratively the linear momentum equations for the increment of nodal velocities $\Delta \bar{u}$ and the continuity equation for the nodal pressures $\overline{\boldsymbol{p}}$ (variables marked with the upper symbol $\overline{\boldsymbol{}}$ refer to nodal values). Once the converged nodal velocities and pressures are obtained, the next time step is considered. At the beginning of the new step, the mesh quality is checked. Whenever an element reaches a distortion level higher than a prearranged limit, a new mesh is rebuilt following the PFEM remeshing procedure explained in Section 3.1.

The solution scheme is summarized for a generic time step $\left[t^{n} ; t^{n+1}\right]$ of duration $\Delta t$ in the pseudo-code given in Algorithm 1.

All matrices and vectors in Algorithm 1 are defined in Appendix A.

Remark 4 The procedure by which one obtains approximations to the derivatives of a field, given an approximation of the field itself, is generally called derivative recovery [37]. In our work, the derivatives of the fluid velocity are needed to calculate several terms in Eq.(8). The most important of these is the material derivative $\frac{\mathrm{D} \boldsymbol{u}}{\mathrm{D} t}$ involved in Eq.(9) and Eq.(10). It turns out that Lagrangian methods such as PFEM have an advantage over traditional Eulerian methods in this respect, as the material derivatives can be calculated simply as finite differences in time, as all quantities are convected along with the flow. This circumvents the need to calculate spatial derivatives of the solution (e.g., the velocity gradient), which are typically of lower-order accuracy due to numerical differentiation. 


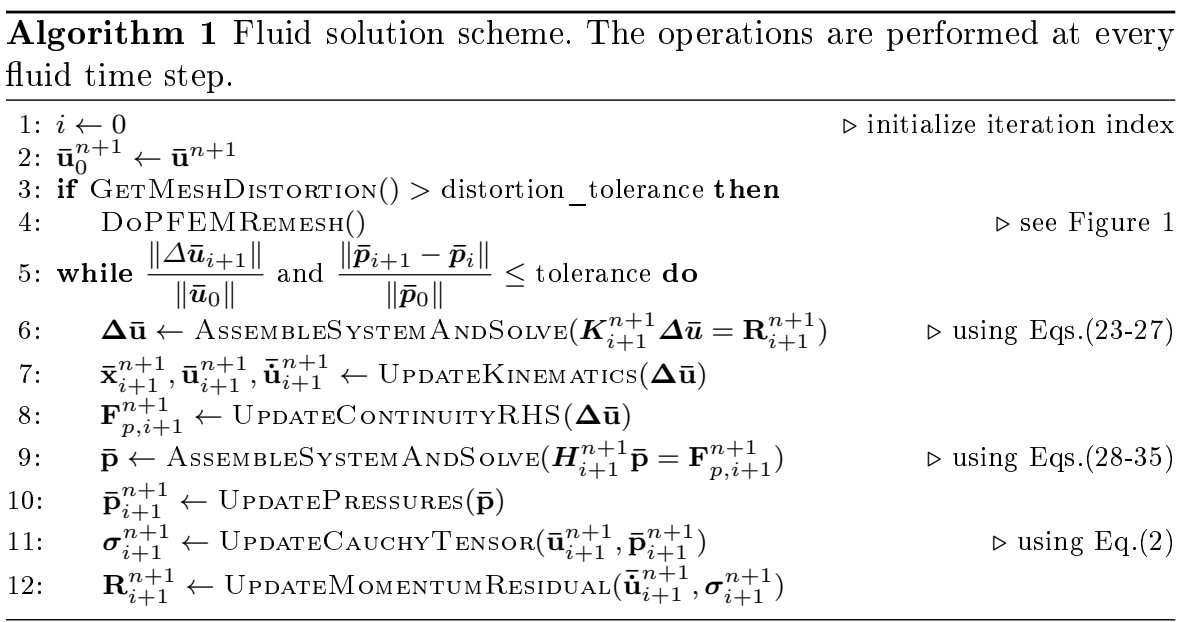

\subsection{Fluid remeshing in PFEM-DEM}

Unlike the PFEM-DEM formulation presented in [39,40], the PFEM remeshing is here insensitive to the solid particles, which are allowed to move freely across the finite element boundaries. This is beneficial to the quality of the discretization since it unlinks the density and location of the fluid nodes from the motion of the particles.

The PFEM integrates the governing equations over the discretized domain as in the standard FEM and the mesh nodes are moved according to the governing equations solution. In large deformation processes, as the ones considered in this work, the mesh nodes motion deteriorates inevitably the discretization (Figure 2b). Hence, in order to preserve a good FEM mesh, the quality of the tessellation is checked at the beginning of each time step (Algorithm 1). If the level of distortion is higher than a fixed threshold, the mesh is deleted and a new discretization is built over the same nodes' positions of the previous mesh (Figure 1).

Remark 5 In order to avoid undesirable concentrations or rarefactions of mesh nodes, some nodes may be removed and/or inserted before the elimination of the previous mesh (Figure 1a). In such case, the nodal values of the new points are obtained by interpolation from neighbouring nodes' values.

The remeshing procedure is one of the most characteristic features of the PFEM. First, all the elements of the distorted mesh are disregarded and all the problem information is stored at the nodes (Figure 1a). Then, a new mesh is generated by performing a Delaunay triangulation [15]. Finally, using the Alpha Shape method [14], the actual boundaries of the computational domain are identified by removing the most distorted elements of the mesh (Figure 1c). This is done by erasing all those elements that do not fulfill the following relation

$$
R<\alpha h
$$


where $R$ is the circumradius of the element and $h$ is the characteristic mesh size. In $2 \mathrm{D}$, the parameter $\alpha$ is often taken as 1.25 . For $3 \mathrm{D}$ problems a slightly higher value is generally chosen. A review of the PFEM remeshing process is given in $[4,2,20]$.
(a) Erase elements
(b) Delaunay Triangulation
(c) Alpha Shape method
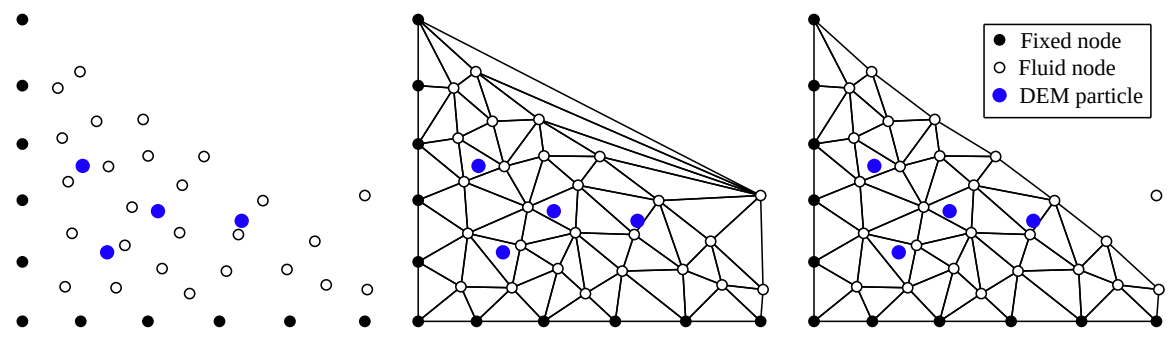

Fig. 1: Scheme of the PFEM-DEM remeshing algorithm.

\section{Particles solution scheme}

The dynamics of the particles are solved with the standard DEM [41,39]. The fact that the particles are submerged in the fluid only affects them in the form of additional forces in their equations of motion (see Eq.(6)).

The typical algorithm for solving the DEM equations consists in the steps listed in Algorithm 2. In the pseudo-code, the sub-index $p$ loops over all particles, while sub-index $k$ loops over the neighbors of each particle.

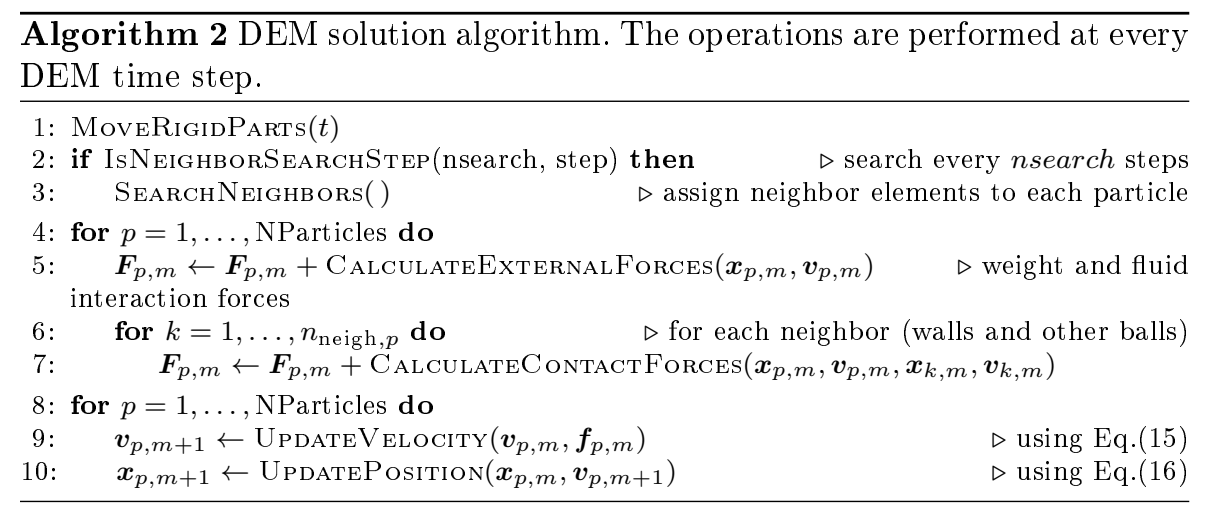

Remark 6 The neighbors search is a very expensive operation, hence it is common to skip this search during some time steps, provided that the neighbors 
found at the last call are generally still valid (the search is done in an extended radius for safety reasons).

The contact forces between particles and between particles and walls follow the formulation detailed in [38], consisting in a Hertz contact model with Coulomb's friction and a viscous damper.

The position and velocity updates can be carried out following any of the available integration schemes in the literature. In our case, the explicit Symplectic Euler scheme [64] has been used, which can be expressed as

$$
\begin{gathered}
\boldsymbol{v}_{p, m+1}=\boldsymbol{v}_{p, m}+\Delta t_{D E M} \frac{\boldsymbol{f}_{p, m}}{m_{p}} \\
\boldsymbol{x}_{p, m+1}=\boldsymbol{x}_{p, m}+\Delta t_{D E M} \boldsymbol{v}_{p, m+1}
\end{gathered}
$$

where sub-index $m$ corresponds to the current configuration, and $m+1$ corresponds to the updated configuration.

\section{PFEM-DEM coupling algorithm}

In the previous sections, the governing equations and the solution algorithms for the fluid phase and the particles phase have been presented separately. In this section, we briefly explain how the two parts interact with each other.

As stated in Section 1, the focus of the present paper is on the one-way coupled PFEM-DEM interaction problem. In our case, the fluid solution affects the movement of the disperse phase (forward coupling), whereas the effect of particles motion on the fluid solution (backward coupling) is not considered. In this work, we apply an updated-fluid strategy, in which the continuum fluid phase is solved first (Figures $2 \mathrm{a}$ and $2 \mathrm{~b}$ ), and then the motion of the suspended particles is calculated using the results of the former (Figure 2c).

$$
\text { (a) } t=t^{n}
$$

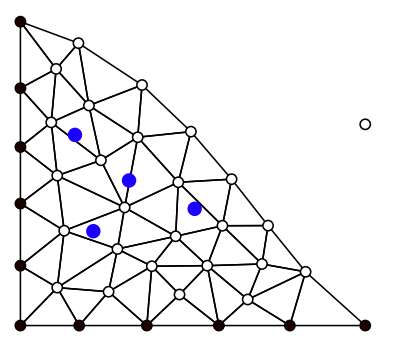

(c) $t=t^{n+1}$. Solve DEM

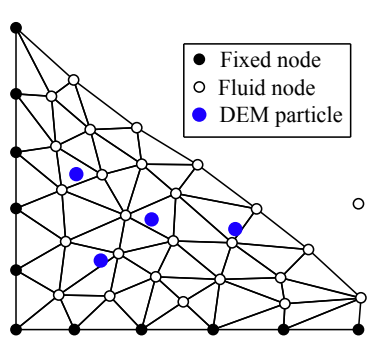

Fig. 2: Scheme of the PFEM-DEM algorithm.

In order to apply the forward coupling algorithm, it is necessary to determine the fluid variables at the particles' position. This process involves 
two steps: first, locate the relevant fluid elements for each particle, and then compute the variables of interest at the particles' position from the selected elements.

The first step is achieved by an efficient search algorithm. For this purpose, a bin data structure [42] containing all the elements of the background mesh and all the discrete particles is created at each search step. Knowing the cell where the particle is placed, the number of the possible neighbor elements is limited to a relatively small amount, thus making this search fast.

Once all the particles are located over the fluid mesh, it is easy to obtain the fluid data at their positions $\boldsymbol{x}_{P}$. For instance, the $i$-component of the fluid velocity at the position of a particle $u_{i}\left(\boldsymbol{x}_{P}\right)$ is evaluated as

$$
u_{i}\left(\boldsymbol{x}_{P}\right)=N^{b n}\left(\boldsymbol{x}_{P}\right) \overline{\boldsymbol{u}}_{i}^{b n}
$$

where $N^{b n}\left(\boldsymbol{x}_{P}\right)$ is the linear shape function evaluated at the position of the particle $P$ and $b n$ ranges from 0 to the number of nodes of the fluid element containing the particle.

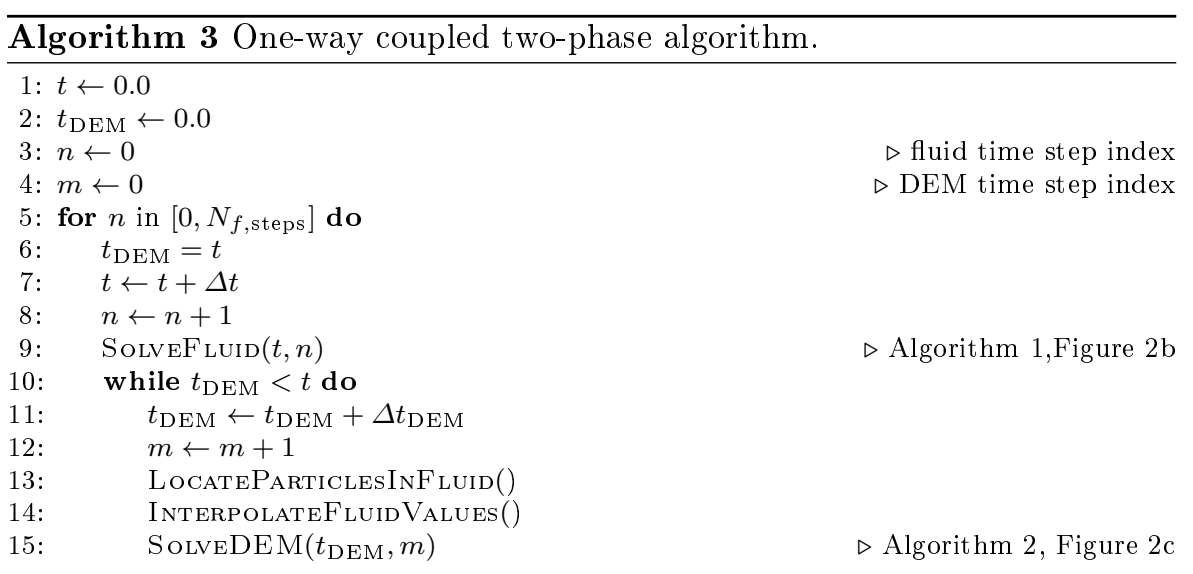

Algorithm 3 shows the sub-stepping associated to forward coupling. Indeed, since the two coupled phases tend to have very distinct characteristic scales, it is often desirable to work with different time integration schemes. The particles integration can be performed with just one step per fluid step, but typically more sub-steps are required.

\section{Validation examples}

In this section, four validation tests for particle-laden flows are studied.

In the first one, the sedimentation of a solid sphere into a tank filled with water is analyzed. The numerical solution is compared to the analytical one given by Stoke's law. 
The second test considers again the sedimentation of a single spherical particle in a water reservoir, but in this case, the container moves upward with a velocity equal to the analytical terminal velocity of a settling particle. In these conditions, the particle is expected to remain still after a brief transient phase. This test is also used to study the effect of the PFEM remeshing on the PFEM and DEM solutions.

The third example consists of a water dam-break test with six embedded particles. The problem aims to validate the fluid phase, but it is also proposed as a benchmark solution of the spherical particles motion for future comparisons with other CFD-DEM solvers.

Finally, the discharge of a tank containing a collection of suspended particles is modeled. The motion of the fluid is compared to known analytical solutions and the possibility of using the solid particles solved with the DEM as tracers of the fluid motion is also investigated.

\subsection{Sedimentation of a sphere in a fluid at rest}

The first test case simulates the sedimentation of a rigid sphere within a static volume of water. This problem is one of typical benchmark tests for CFDDEM solvers [21-23]. The particle falls under the effect of gravity and the steady viscous resistance. For small particle Reynolds number, the motion of the particle is well approximated by the standard Stoke's law. As in [21], the time variation of the particle vertical velocity is computed analytically as

$$
v_{y}=\frac{g V_{p}\left(\rho_{p}-\rho_{f}\right)}{3 \pi \mu_{f} d_{p}}\left(1-e^{\frac{-3 \pi \mu_{f} d_{p} t}{V_{p} \rho_{p}}}\right)
$$

where $g=9.81 \mathrm{~m} / \mathrm{s}^{2}$ is the gravity acceleration, $V_{p}$ is the volume of the spherical particle, $\rho_{p}=2500 \mathrm{~kg} / \mathrm{m}^{3}$ and $\rho_{f}=1500 \mathrm{~kg} / \mathrm{m}^{3}$ are the particle and water densities, respectively, $\mu_{f}=0.001 \mathrm{~Pa} \cdot \mathrm{s}$ is the water viscosity, and $d_{p}=0.1 \mathrm{~mm}$ is the diameter of the particle. Note that this analytical relation is obtained if only the drag force term is considered in Eq.(8) (i.e. $\boldsymbol{F}_{\boldsymbol{h y} \boldsymbol{y} \text { dro }}=\boldsymbol{F}_{\boldsymbol{D}}$ ).

In Figure 3 a schematic representation of the initial geometry and a horizontal section of the PFEM mesh (mean element size $0.15 \mathrm{~m}$ ) are plotted. The particle is placed at the middle of a cylindrical water tank. In all graphical representations, the spherical particle is artificially enlarged for visualization purposes.

At the onset of the simulation, the position of the solid sphere is kept fixed, allowing the fluid to reach a hydrostatic state. After this initialization phase, the particle is let free to fall. The time instant at which the particle is released is considered as the initial time of the numerical simulation $(t=0)$.

In Figure 4 the time evolution of the vertical velocity obtained with the proposed PFEM-DEM approach is plotted together with the analytical one from Eq.(18). 

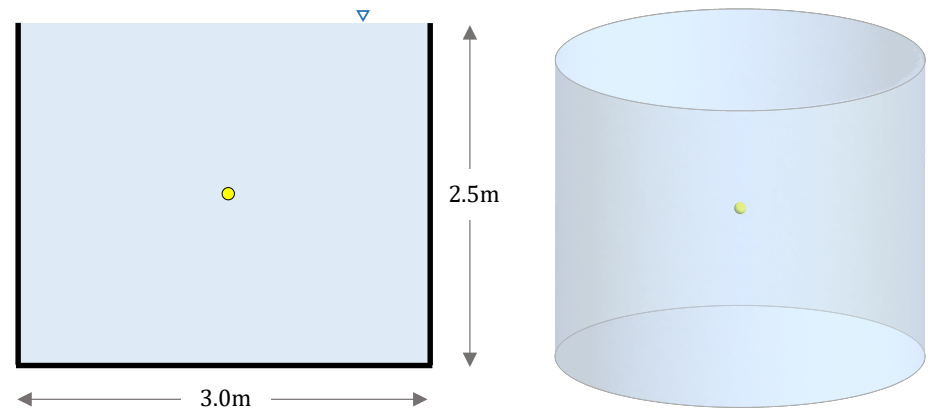

Fig. 3: Sedimentation of a sphere in a fluid at rest. Lateral and $3 \mathrm{D}$ view.

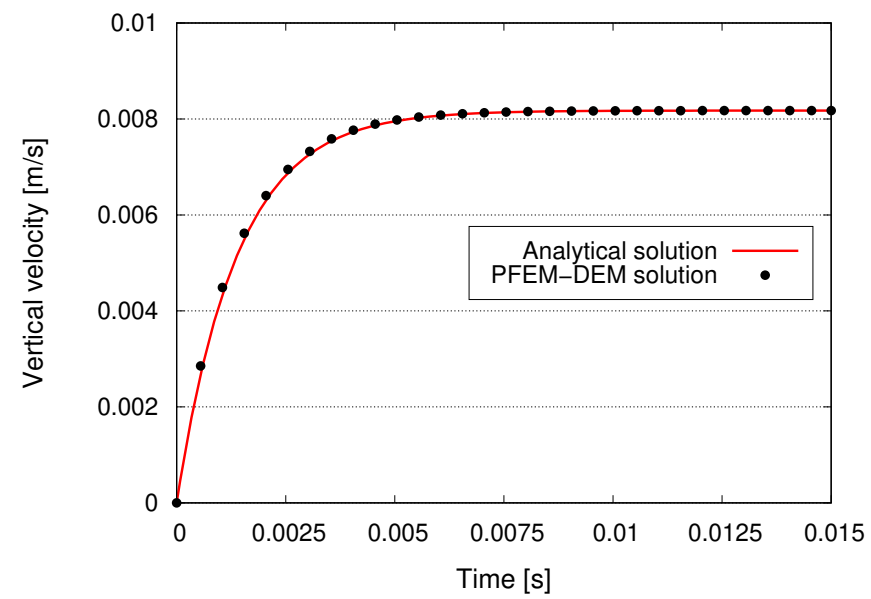

Fig. 4: Sedimentation of a sphere in a fluid at rest. Evolution of particle's vertical velocity (absolute value). PFEM-DEM and analytical (Eq.(18)) solutions.

The graphs show that the numerical solution is almost identical to the analytical prediction, proving that the proposed PFEM-DEM approach fulfills this benchmark test for CFD-DEM solvers.

\subsection{Sedimentation of a sphere in a rising water tank}

This second test has been designed to analyze the PFEM-DEM coupling algorithm in cases where the particle moves through different fluid mesh elements. It is also a suitable example in order to evaluate the effect of remeshing over the global numerical solution accuracy.

For this purpose, the same test analyzed in Section 6.1 is once more considered here with some modifications. The geometry and the material properties are the same as in the previous problem, but, in this case, the fluid boundaries, except the top free-surface contour, are forced to move upwards with a 
constant vertical velocity $\hat{u}_{y}=0.008175 \mathrm{~m} / \mathrm{s}$, this being the terminal velocity of the settling particle as predicted by Stoke's law.

Concerning the spherical particle, its initial vertical velocity has been set equal to $v_{y}=-0.02 \mathrm{~m} / \mathrm{s}$. Under these conditions, the particle is expected to reach a quiescent state while the surrounding fluid keeps moving upwards. A schematic representation of the initial state of the test is shown in Figure 5.

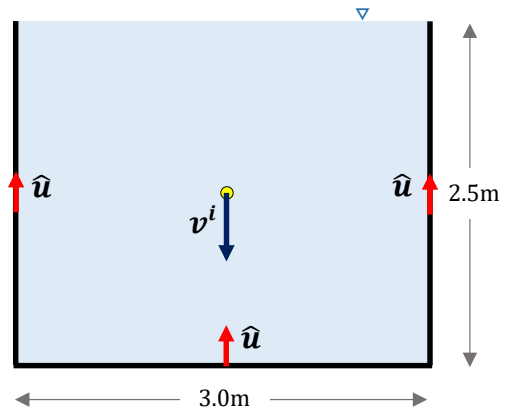

\begin{tabular}{|c|c|c|}
\hline \multicolumn{3}{|c|}{ Fluid boundary velocity $\hat{\boldsymbol{u}}$} \\
\hline$\hat{u}_{x}[\mathrm{~m} / \mathrm{s}]$ & $\hat{u}_{y}[\mathrm{~m} / \mathrm{s}]$ & $\hat{u}_{z}[\mathrm{~m} / \mathrm{s}]$ \\
\hline 0 & 0.008175 & 0 \\
\hline \hline \multicolumn{3}{|c|}{ Particle initial velocity $\boldsymbol{v}^{i}$} \\
\hline \hline$v_{x}^{i}[\mathrm{~m} / \mathrm{s}]$ & $v_{y}^{i}[\mathrm{~m} / \mathrm{s}]$ & $v_{z}^{i}[\mathrm{~m} / \mathrm{s}]$ \\
\hline 0 & -0.02 & 0 \\
\hline
\end{tabular}

Fig. 5: Sedimentation of a sphere in a rising water tank. Initial geometry scheme and boundary conditions (lateral view).

Figure 6 shows three snapshots of the particle at three consecutive time instants of the initial phase of the test. The portion of mesh shown in the pictures corresponds to a vertical section of the domain passing through the particle's center. The computed velocity vectors are plotted over the fluid mesh and on the solid particle. The sequence shows that the particle decelerates quickly and reaches a steady state while the surrounding fluid keeps moving upwards.

(a) $\mathrm{t}=0 \mathrm{~s}$

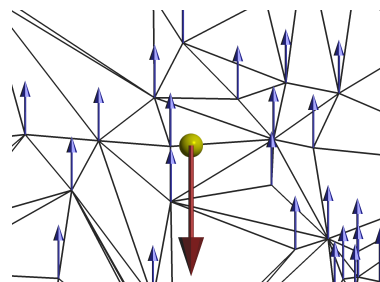

(b) $\mathrm{t}=0.001 \mathrm{~s}$

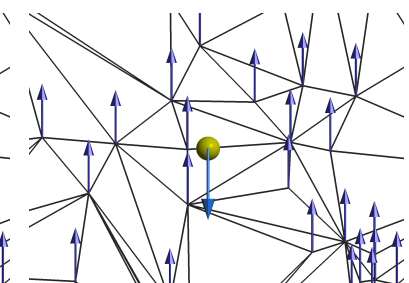

(c) $\mathrm{t}=0.004 \mathrm{~s}$

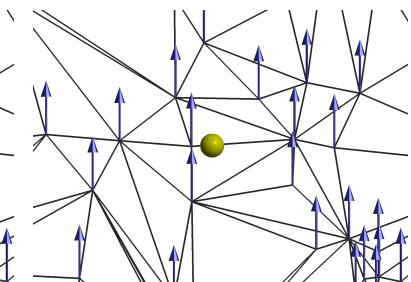

Fig. 6: Sedimentation of a sphere in a rising water tank. Velocity vectors plotted over the central zone of a vertical section at three different time instants.

This behavior is quantitatively represented in the graph of Figure 7, where the time evolution of the vertical velocities of both the particle $(\boldsymbol{v})$ and the fluid evaluated at the particle's center $\left(\boldsymbol{u}\left(\boldsymbol{x}_{\boldsymbol{p}}\right)\right)$ are plotted. The graphs show 
that the fluid moves with a constant velocity (almost) equal to that imposed over the rigid boundaries, while the spherical particle decelerates and asymptotically approaches an (almost) null velocity, as expected.

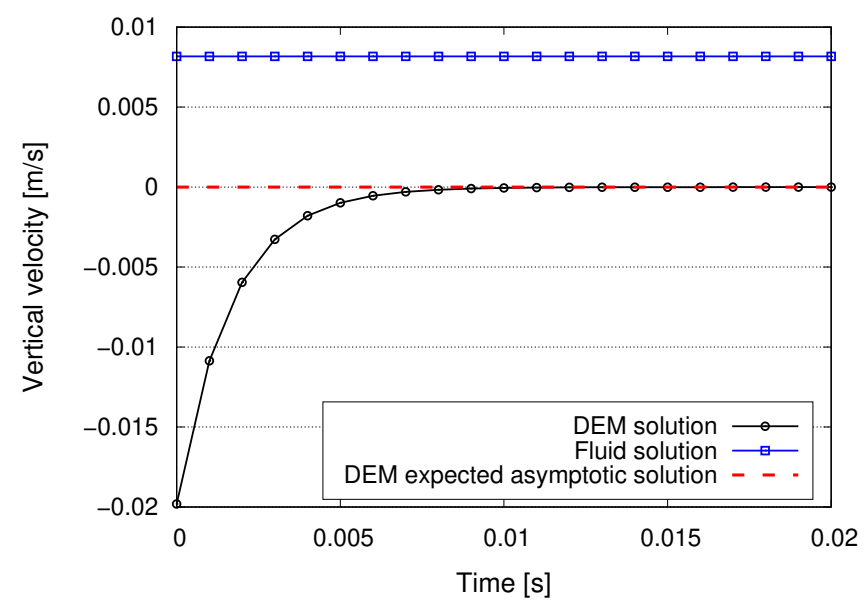

Fig. 7: Sedimentation of a sphere in a rising water tank. Time evolution of the fluid vertical velocity evaluated at the particle's center $\left(u_{y}\left(\boldsymbol{x}_{\boldsymbol{p}}\right)\right)$ and particle's vertical velocity $\left(v_{y}\right)$.

This test is also used to investigate the effects related to the PFEM remesh algorithm. It is expected that the PFEM mesh regeneration affects the fluid solution and, consequently, also the DEM one. However, it is interesting to get a quantitative reference for this perturbation. For this purpose, two cases are studied. In the first one, the same mesh is maintained unchanged for all the duration of the analysis. Instead, in the second one, the mesh is modified at $t=2 s$ by adding a new mesh node within the fluid element that contains the spherical particle.

Figure 8 shows the velocity results obtained at $t=2 s$ with and without remeshing. Judging from the appearance, the two solutions seem to be identical, despite the change of the discretization.

However, in reality, the PFEM-DEM solution is affected by the change in the mesh. In order to appreciate this perturbation, in Figure 9 the time evolution of the fluid and particle velocities is re-plotted considering a very small range of velocity values.

At this resolution, it appears clearly that the mesh variation induces a jump on the PFEM results. However, this perturbation is small and it is of the order of magnitude of the error of the PFEM solution (around 0.015\%). After the shock, the perturbed solution computed after remeshing tends to recover the one obtained without remeshing. Figure $9 \mathrm{~b}$ shows that this behavior is inherited by the DEM solution. In fact, the particle's velocity exactly mir- 
(a) No remeshing

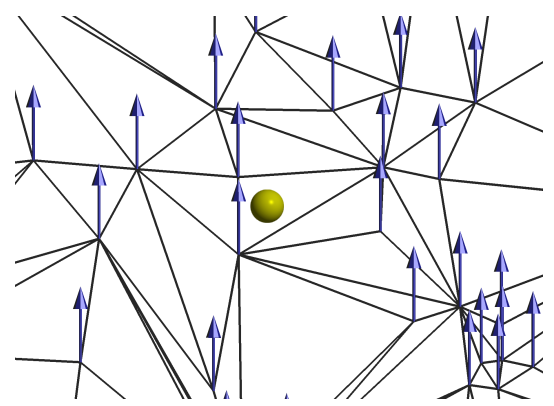

(b) Remesh at $\mathbf{t}=2 \mathrm{~s}$

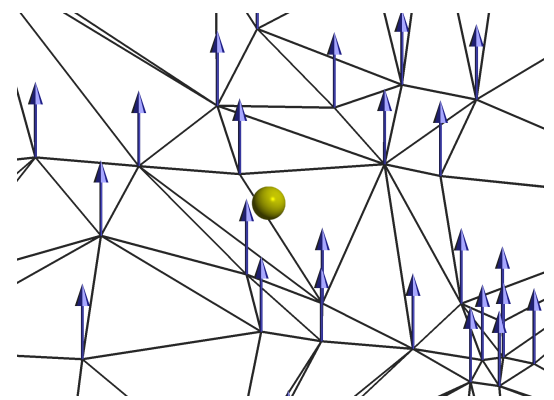

Fig. 8: Sedimentation of a sphere in a rising water tank. Velocity vectors over at $t=2 \mathrm{~s}$. (a) The initial mesh is maintained. (b) A mesh node has been added within the element containing the spherical particle.

rors the fluid solution, highlighting the efficiency of the DEM-PFEM coupling scheme.

These results suggest that, although the generation of a new tessellation inevitably induces a perturbation of the FEM solution, this shock has a limited effect on the numerical results and it is of the same order of magnitude as the solution error.

Nevertheless, it should be emphasized that this analysis focused on a localized change of tessellation affecting only an inner part of the fluid domain. For more massive variations of the connectivity of the elements, and/or when the generation of a new mesh also induces changes in the fluid topology (e.g. creation or elimination of boundary elements), the perturbation of the numerical results can be larger. In particular, in these cases, volume conservation associated with remeshing is the most critical issue. This problem is studied in detail in [20]. In this reference, it is confirmed that the PFEM remeshing may induce spurious variations of the fluid volume, however, it is also shown that these volume changes can be limited with a proper selection of the $\alpha$ parameter of the Alpha Shape method and they become almost negligible when a fine mesh is used.

\subsection{Water dam break with embedded particles}

The water dam break test [24-26] is frequently used to validate free-surface fluids solvers [27-30]. In this section, this problem is presented to validate the fluid dynamics solver of the proposed PFEM-DEM strategy. The test consists in the collapse of a water column caused by the removal of the vertical retaining wall.

In contrast with the standard water dam break, six spherical particles are here initially placed within the water column. We note that the solid particles 
(a) Fluid solution

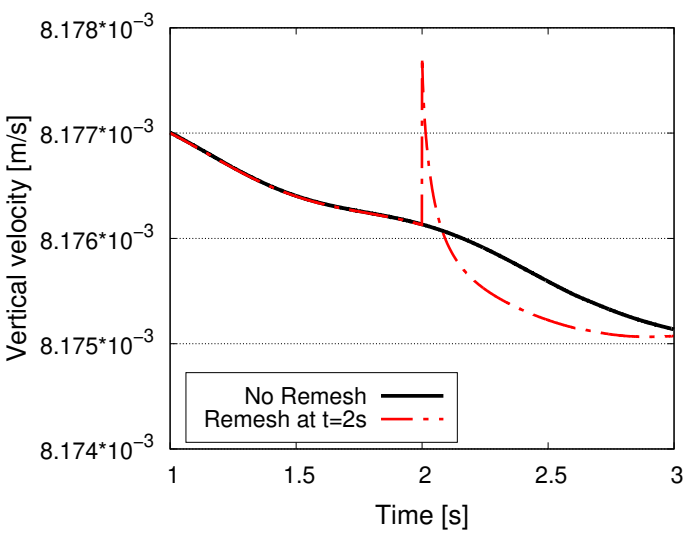

(b) DEM solution

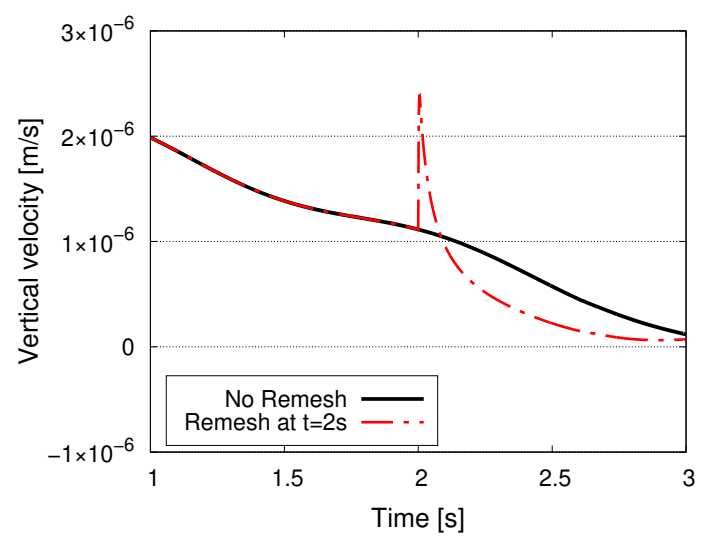

Fig. 9: Sedimentation of a sphere in a rising water tank. (a) Time evolution of the fluid vertical velocity evaluated at the particle's center $\left(u_{y}\left(\boldsymbol{x}_{p}\right)\right)$. (b) Time evolution of particle's vertical velocity $\left(v_{y}\right)$. Results for the time interval $[1 s, 3 s]$.

are small and dispersed, and so their effect on the fluid solution can be safely neglected (one-way coupling).

The initial geometry of the test, the fluid mesh and material data are given in Figure 10. All the solid particles are assumed to be identical spheres with radius $r_{p}=0.75 \mathrm{~mm}$ and density $\rho_{p}=1500 \mathrm{~kg} / \mathrm{m}^{3}$. The coordinates of their initial positions are given in Figure 11.

In Figure 12 the numerical results for the fluid problem are compared qualitatively to those obtained experimentally in [25] for the same time instants.

For this test, a quantitative validation of its initial phase is also possible. In particular, before the impact of the water against the vertical wall, it is possible to plot the advancing in time of the fluid front and compare it to experimental 


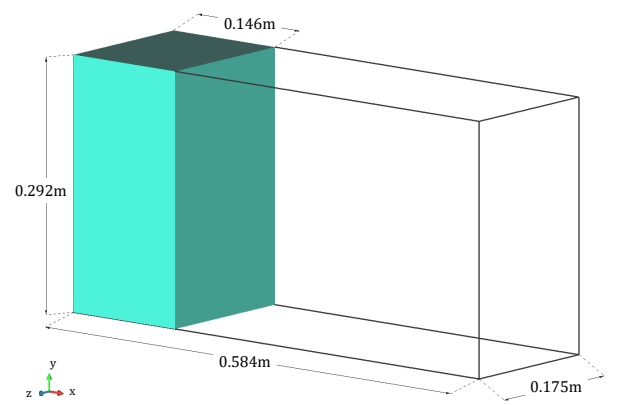

\begin{tabular}{|l|c|}
\hline \multicolumn{2}{|c|}{ Fluid material data } \\
\hline \hline Density $\left[\mathrm{kg} / \mathrm{m}^{3}\right]$ & 1000 \\
\hline Bulk modulus $[\mathrm{Pa}]$ & $2.1 \cdot 10^{9}$ \\
\hline Viscosity $[\mathrm{Pa} \cdot \mathrm{s}]$ & 0.001 \\
\hline \multicolumn{2}{|c|}{ Fluid mesh data } \\
\hline \# of tetrahedra & 753303 \\
\hline Mean mesh size [mm] & 4.63 \\
\hline
\end{tabular}

Fig. 10: Water dam break with embedded particles. Initial geometry and fluid phase data.

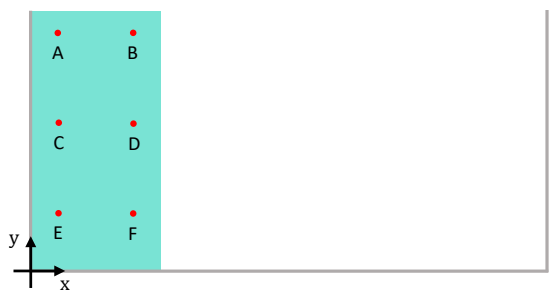

\begin{tabular}{|c|c|c|c|}
\hline Particle & $\mathrm{x}[\mathrm{m}]$ & $\mathrm{y}[\mathrm{m}]$ & $\mathrm{z}[\mathrm{m}]$ \\
\hline \hline A & 0.030 & 0.280 & 0.0885 \\
\hline B & 0.116 & 0.280 & 0.0885 \\
\hline C & 0.030 & 0.200 & 0.0885 \\
\hline D & 0.116 & 0.200 & 0.0885 \\
\hline E & 0.030 & 0.120 & 0.0885 \\
\hline F & 0.116 & 0.120 & 0.0885 \\
\hline
\end{tabular}

Fig. 11: Water dam break with embedded particles. Initial position of DEM spheres.

and numerical results from the literature. Specifically, the 3D numerical results taken as reference are from [28] and [30], where a Moving Particle Semi-implicit method (MPS) and an explicit PFEM were used, respectively.

As in the above references, the following dimensionless variables are considered:

$$
\begin{aligned}
& x^{*}=x / d \\
& y^{*}=y / d \\
& t^{*}=t(2 g / d)^{0.5}
\end{aligned}
$$

where $x$ is the horizontal coordinate (at the beginning $x=0.146 \mathrm{~m}$ for the fluid front), $d=0.146 \mathrm{~m}$ is the base of water column, $y$ is the vertical coordinate (for the fluid front $y=0 \mathrm{~m}$ always), and $g=9.81 \mathrm{~m} / \mathrm{s}^{2}$ is the gravitational acceleration.

In Figure 13 three representative images of the first phase of this numerical test are given.

In Figure 14 the wave front evolution obtained with the PFEM algorithm here proposed is compared to literature results.

The graph shows that all the numerical results (solid lines) are in good agreement, although they all slightly over-predict the fluid front advancing obtained experimentally (discontinuous lines). This is probably due to a not faithful modeling of the retaining wall removal. As pointed out in [30], in all the referenced numerical models the wall is removed instantaneously, while in the laboratory experience this process takes inevitably a finite time. 
(a) $\mathrm{t}=0 \mathrm{~s}$, Exp. from $[25]$

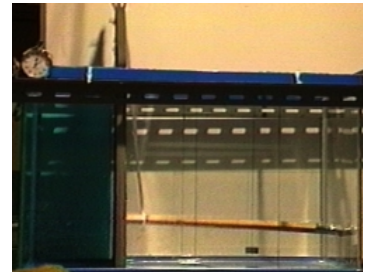

(d) $\mathrm{t}=0 \mathrm{~s}$, PFEM

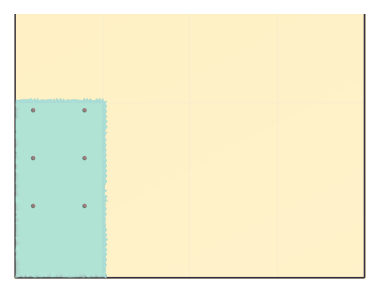

(g) $\mathrm{t}=0.6 \mathrm{~s}$, Exp. from $[25]$

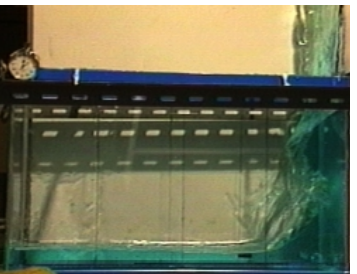

(j) $\mathrm{t}=0.6 \mathrm{~s}$, PFEM

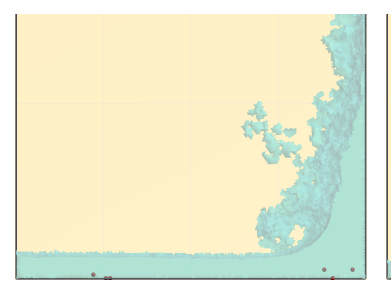

(c) $\mathrm{t}=0.4 \mathrm{~s}$, Exp. from $[25]$

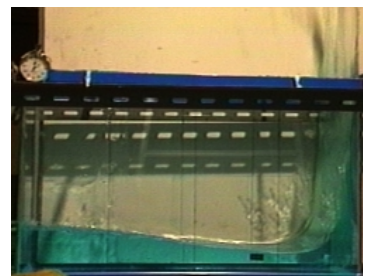

(f) $\mathrm{t}=0.4 \mathrm{~s}, \mathrm{PFEM}$

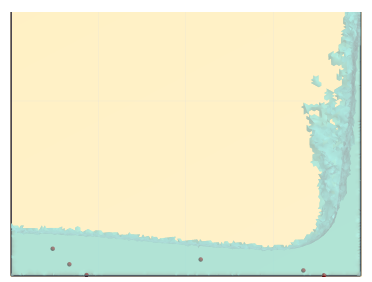

(i) $\mathrm{t}=1.0 \mathrm{~s}$, Exp. from [25]

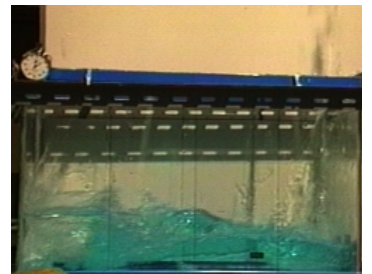

(1) $\mathrm{t}=1.0 \mathrm{~s}$, PFEM

Fig. 12: Water dam break with embedded particles. Numerical and experimental [25] results at the same time instants.

For the same time interval considered in Figure 14, the time evolution of the positions of the six spherical particles is given in Figure 15 with the aim of allowing future comparisons with other CFD-DEM solvers. Only the vertical and horizontal displacements are plotted since, during the considered time interval, the particles undergo a negligible traversal motion. 

(a) $t^{*}=1.05$
(b) $t^{*}=1.97$
(c) $t^{*}=2.90$

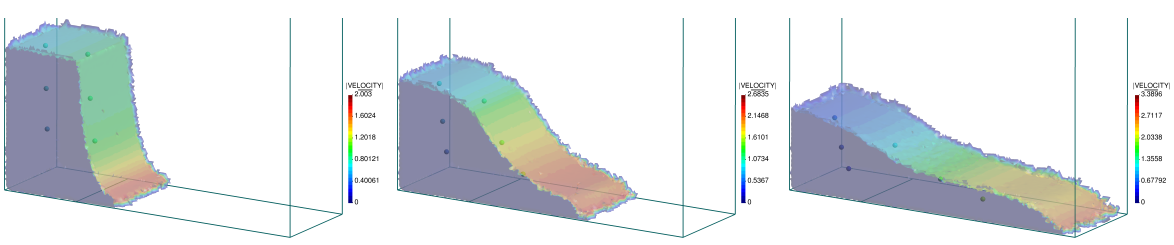

Fig. 13: Water dam break with embedded particles. 3D results with velocity contours plot at three time instants.

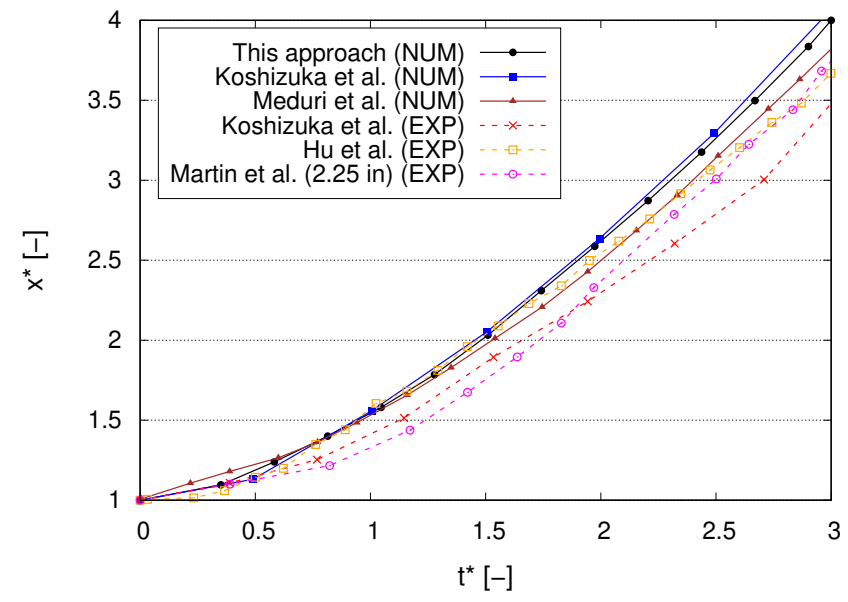

Fig. 14: Water dam break with embedded particles. Dimensionless time evolution of the front wave position. Numerical (NUM) results from Koshizuka et al. [28] (3D MPS) and Meduri et al. [30] (explicit 3D PFEM). Experimental (EXP) results from Koshizuka et al. [25], Hu et al. [26] and Martin et al. [24].

\subsection{Tank discharge}

In this last test, the discharge of a tank containing water and a collection of spherical particles is studied. The container is a cube with sides of $1 \mathrm{~m}$ and an orifice of diameter $D=0.2 \mathrm{~m}$ in the center of its bottom surface. Initially, the water $\left(\rho_{f}=1000 \mathrm{~kg} / \mathrm{m}^{3}, \mu_{f}=0.001 P a \cdot s\right)$ fills the tank entirely. The collection of particles has a cylindrical shape of height $0.1 \mathrm{~m}$ and diameter $0.8 \mathrm{~m}$, and it is formed by 2552 identical spheres of diameter $d_{p}=0.005 \mathrm{~m}$, irregularly distributed within the cloud. The particles are so small and dispersed that it can be assumed that their motion does not affect the fluid flow (one-way coupling). In Figure 16 the initial geometry of the numerical test is given. All the lengths and distances are defined with respect to the orifice diameter $D$.

The same problem is studied for three different values of the particles density, namely $\rho_{p}=1000 \mathrm{~kg} / \mathrm{m}^{3}, \rho_{p}=1125 \mathrm{~kg} / \mathrm{m}^{3}$ and $\rho_{p}=1500 \mathrm{~kg} / \mathrm{m}^{3}$. 
(a) Horizontal position

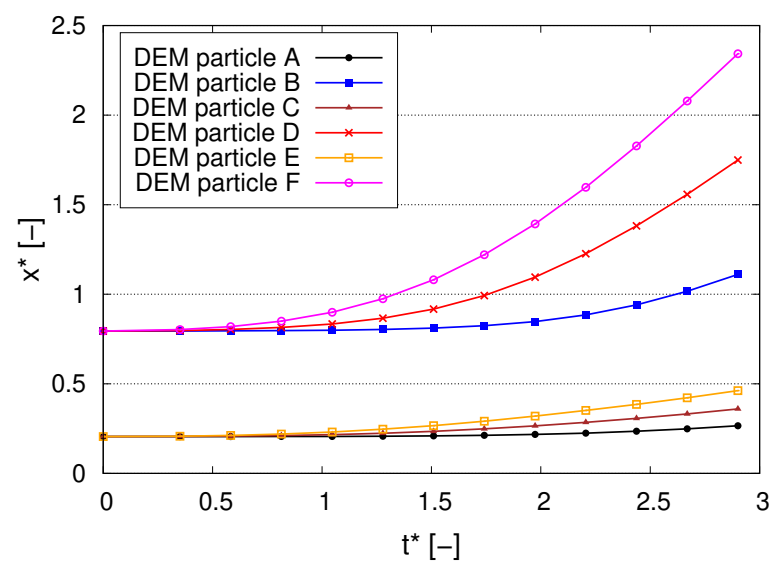

(b) Vertical position

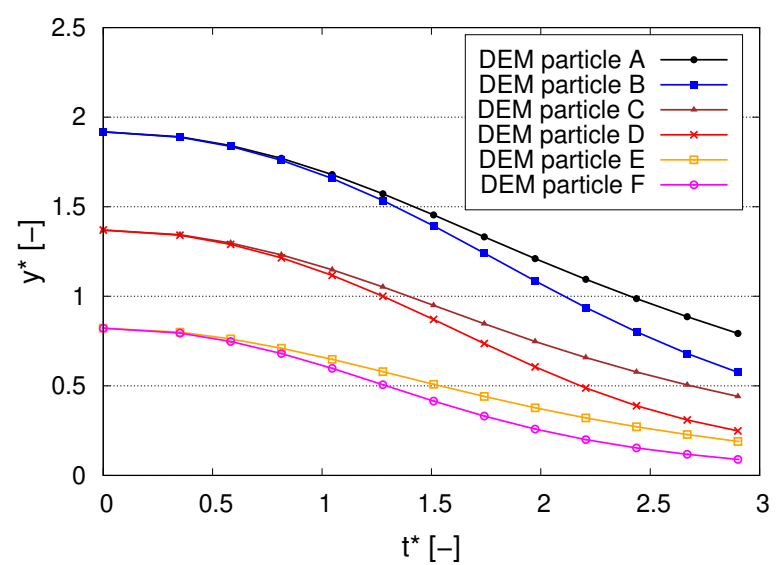

Fig. 15: Water dam break with embedded particles. Dimensionless time evolution of the dimensionless coordinates of spherical particles.

Note that for $\rho_{p}=1000 \mathrm{~kg} / \mathrm{m}^{3}=\rho_{f}$ it is expected that the particles follow exactly the fluid streamlines obtained by the PFEM solution.

A particle placed at the center of the fluid tank at $y=0.65$, is chosen as the reference of the particles motion in the three cases. In the following, this particle will be referred to as the sample particle. The initial fluid mesh is made of 879209 tetrahedral elements, with a mean size equal to $h=0.0225 \mathrm{~m}$. 
(a) Lateral view

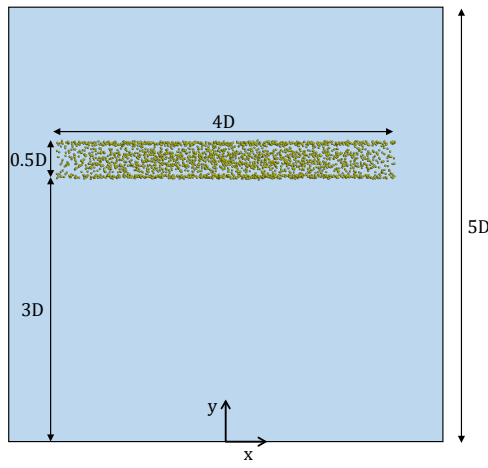

(b) View from the top

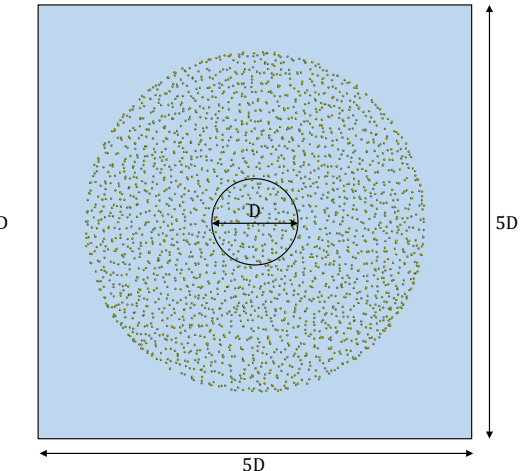

Fig. 16: Tank discharge. Initial geometry $(D=0.2 m)$.

For convenience, the following dimensionless variables are used

$$
\begin{aligned}
& y^{*}=y / D \\
& v^{*}=a b s\left(v_{y}\right) /(2 g H)^{0.5} \\
& t^{*}=t(2 g H)^{0.5} / D \\
& \rho^{*}=\rho_{p} / \rho_{f} \\
& M^{*}=M_{f} / M_{f i}
\end{aligned}
$$

where $y^{*}, v^{*}, t^{*}, \rho^{*}$ and $M^{*}$ are the dimensionless vertical coordinate, velocity, time, particle density and water mass, respectively. Furthermore, $H=1 \mathrm{~m}$ is the initial water level, $g=9.81 \mathrm{~m} / \mathrm{s}^{2}$ is the gravity, $M_{f}$ is the water mass contained in the tank and $M_{f i}=1000 \mathrm{~kg}$ its initial value.

Figure 17 shows a sequence of six snapshots obtained from the numerical simulation, for $\rho^{*}=1.125$. The figures show that the fluid exits through the orifice forming a stream with a reduced cross-section with respect to the hole, the characteristic vena contracta. A flat free-surface is maintained above. This configuration is kept by the fluid for the entire duration of the test $\left(t^{*}=225\right)$.

Turning to the particles, the initial cloud begins settling with near-uniform velocity, thus keeping its shape almost unaltered (Figure 17a). However, when the solid particles approach the orifice (approximately at a distance equal to the diameter of the hole), the particles suffer a sudden acceleration toward the center of the tank (Figures 17b-17d). We note that among the outermost particles in the cloud, those placed at a larger distance from the lateral walls move faster towards the orifice. This motion alters the initially circular shape of the cloud. At around $t^{*}=77.5$, the outermost particles in the cloud start hitting the lower surface of the tank (Figures 17e-17f) and they are progressively dragged to the orifice.

Similar behavior is observed for $\rho^{*}=1.5$, although the sedimentation of the particles is faster. On the other hand, for $\rho^{*}=1$ the particles, as expected, follow the fluid streamlines and none of these impacts the lower wall of the 
(a) $t^{*}=33.2,3 \mathrm{D}$ and top view

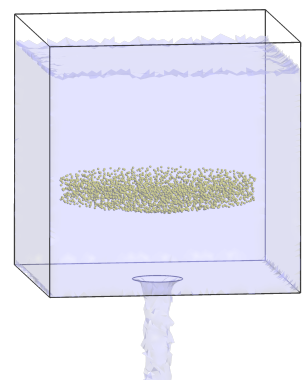

(c) $t^{*}=55.4,3 \mathrm{D}$ and top view

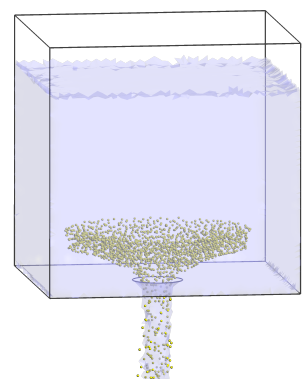

(e) $t^{*}=77.5,3 \mathrm{D}$ and top view
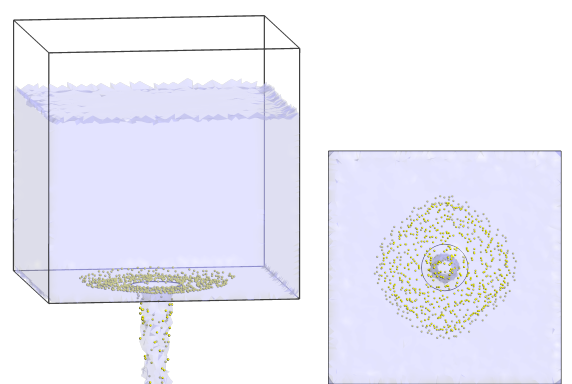

(b) $t^{*}=44.3,3 \mathrm{D}$ and top view
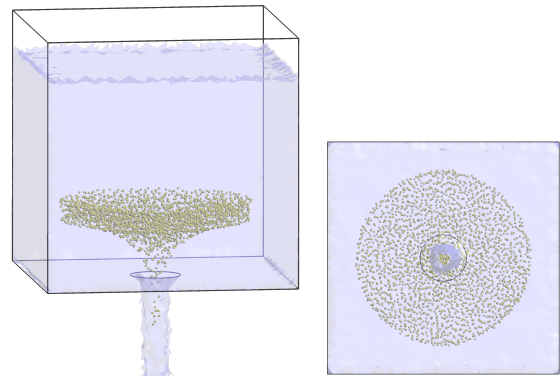

(d) $t^{*}=66.4,3 \mathrm{D}$ and top view

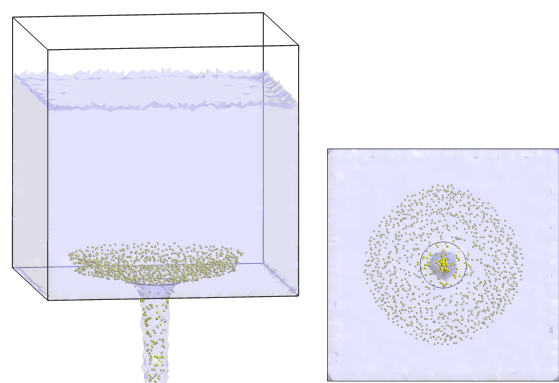

(f) $t^{*}=88.6,3 \mathrm{D}$ and top view
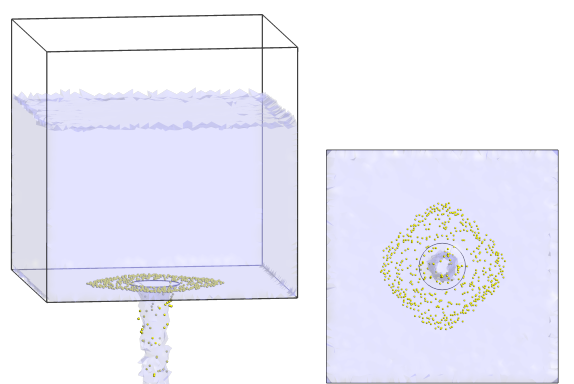

Fig. 17: Tank discharge. Numerical results at six time instants for $\rho^{*}=1.125$.

container. This is clearly shown in Figure 18, where the particle clouds for the three studied densities are overlapped to the fluid streamlines of the central $x z$-section. The cases of $\rho^{*}=1.5$ (Figures 18i-181) and $\rho^{*}=1.125$ (Figures $18 \mathrm{e}-18 \mathrm{~h}$ ) show a clear discrepancy between the particles motion and the fluid streamlines. On the contrary, for $\rho^{*}=1$ (Figures 18a-18d) the particles behave perfectly as tracers of the fluid motion. This represents a further evidence of the accuracy of the proposed PFEM-DEM coupling algorithm. Note also that using spherical particles as tracers can be very useful in a PFEM framework. In fact, in PFEM analyses, the mesh nodes cannot be generally used for this 
purpose because they can be moved, erased or replaced during the remeshing step to maintain the mesh quality (see Remark 5 in Section 3.1).

(a) $\rho^{*}=1.0 t^{*}=11.1$

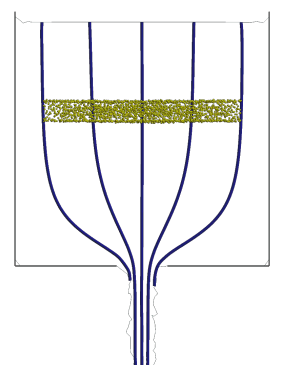

(e) $\rho^{*}=1.125 t^{*}=11.1$

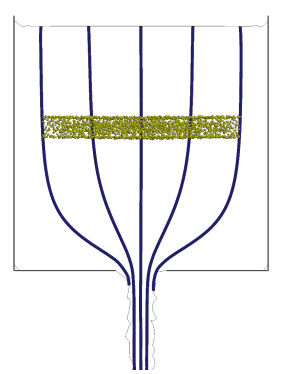

(i) $\rho^{*}=1.5 t^{*}=11.1$

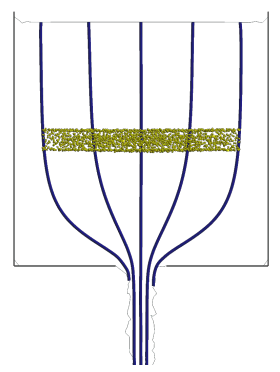

(b) $\rho^{*}=1.0 t^{*}=66.4$

(c) $\rho^{*}=1.0 t^{*}=121.8$

(d) $\rho^{*}=1.0 t^{*}=166.1$
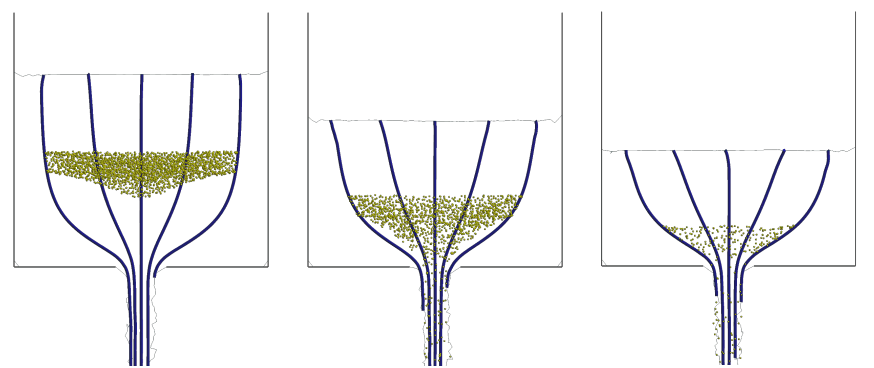

(f) $\rho^{*}=1.125 t^{*}=33.2$

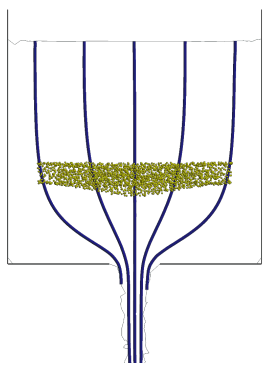

(j) $\rho^{*}=1.5 t^{*}=22.1$

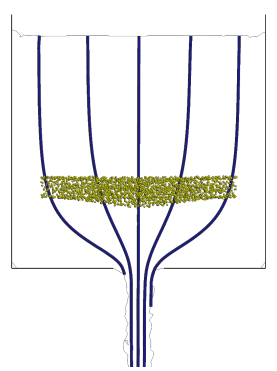

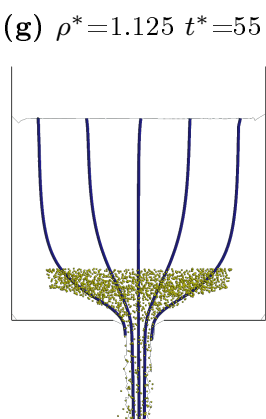

(k) $\rho^{*}=1.5 t^{*}=33.2$

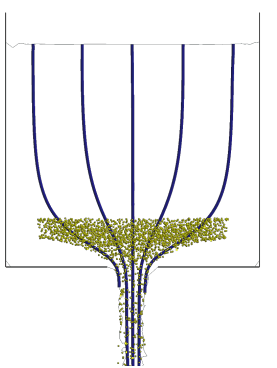

(h) $\rho^{*}=1.125 t^{*}=77.5$

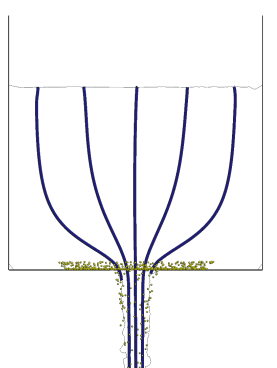

(1) $\rho^{*}=1.5 t^{*}=44.3$

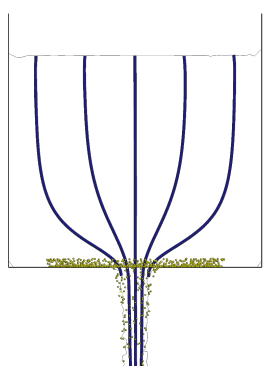

Fig. 18: Tank discharge. Fluid streamline plotted over the central $x z$-section. DEM solution for three different values of $\rho^{*}=1.5$.

Using Bernoulli's theorem, it is possible to verify the accuracy of the fluid solution. According to this theorem, the outlet velocity of an inviscid and incompressible fluid contained in a tank can be computed as

$$
u=\sqrt{2 g h_{f s}+u_{f s}^{2}}
$$


where $h_{f s}$ is the difference in height between the fluid free-surface and the position where the outlet velocity is measured, and $u_{f s}$ is the fluid velocity at the free-surface.

This simple relation is valid only for negligible pressure differences between the free-surface and the point where the velocity is computed, otherwise, also the effect of pressure must be taken into account.

In order to check this issue, in Figure 19 the pressure field is plotted over a central vertical section. The results show a hydrostatic pressure distribution in the regions far from the hole. Near the orifice, one observes a perturbed pressure field region with a characteristic size similar to the hole diameter. On the other hand, inside the stream beneath the hole, at a distance equal to the radius of the orifice $\left(y^{*}=-0.5\right)$, the fluid pressure almost vanishes, as for the superior free surface. Consequently, Eq.(21) can be used safely to estimate the outlet fluid velocity at $y^{*}=-0.5$.

(a) $t^{*}=11.1$

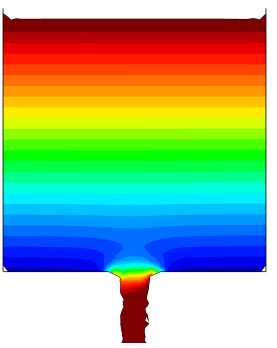

(b) $t^{*}=66.4$

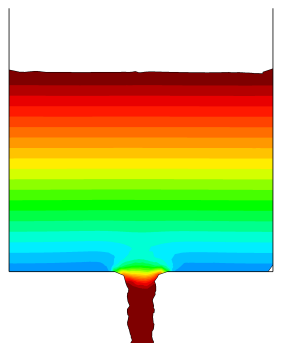

(c) $t^{*}=121.8$

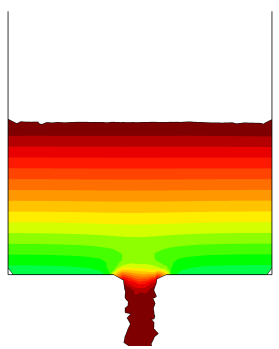

(d) $t^{*}=177.2$

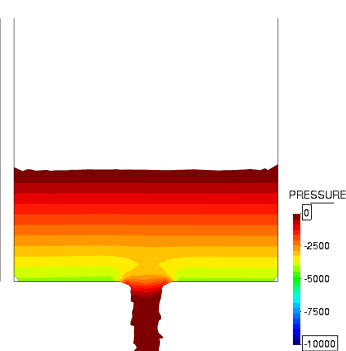

Fig. 19: Tank discharge. Pressure field at the central section $x z$ of the fluid domain.

Figure 20 shows the time evolution of the dimensionless fluid velocity computed at different heights of the central axis $(x=z=0)$. The graphs show that for $y^{*}=-0.5$, the fluid solution agrees well with that predicted by the Bernoulli's theory (Eq.(21)).

It is also interesting to note that starting from $y^{*}=1$, the central part of the fluid undergoes a sudden acceleration. It is remarkable that the jump of velocity between $y^{*}=1$ and $y^{*}=0$ is more than ten times larger than the one computed between $y^{*}=3$ and $y^{*}=1$. This abrupt acceleration is also observed in the dimensionless velocity curve of the sample particle with $\rho^{*}=1.0$, also plotted in Figure 20. At around $y^{*}=1$, the particle suffers a sudden acceleration after having been moving with an almost constant velocity up to that point. When it reaches $y^{*}=-0.5$, the particle decelerates and starts falling with a constant acceleration practically equal to $g$.

This free-fall motion is also exhibited by the other tested sample particles of different densities (see Figure 21b). Note that this occurs when the particle is still contained within the fluid flow $\left(y^{*}=-0.5\right)$, showing that the fluid effect on the DEM solution becomes negligible at a distance equal to the radius of the orifice. 


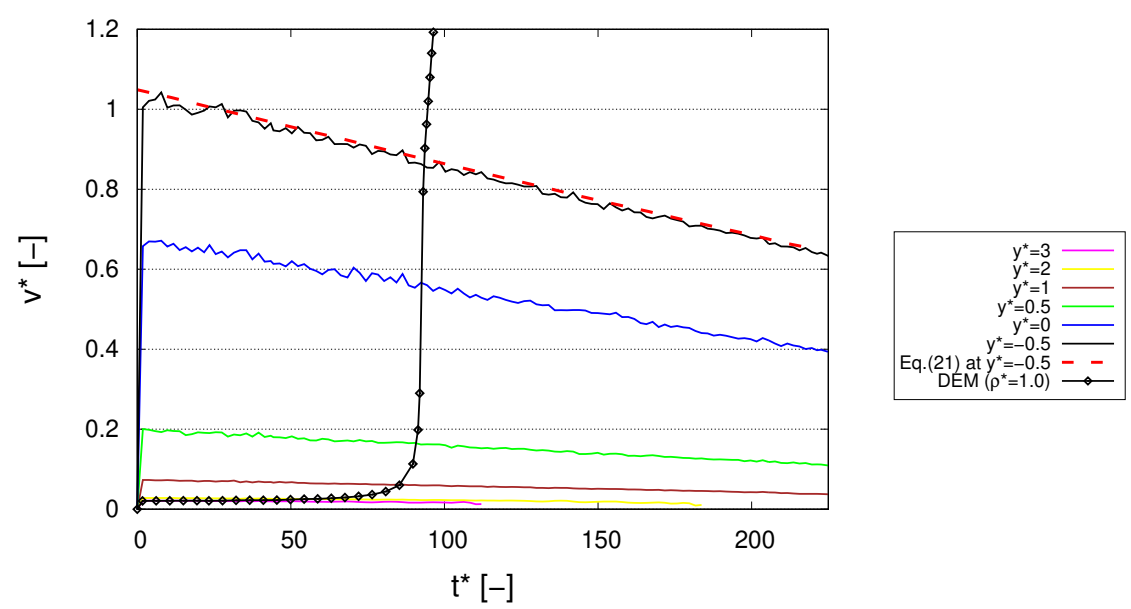

Fig. 20: Tank discharge. Time evolution fo dimensionless velocity. Results at six fluid height (central point), sample particle with $\rho^{*}=1.0$ and theoretical solution according to Eq.(21) at $y^{*}=-0.5$.

The time evolution of the fluid mass leakage through the tank hole can be computed combining the Bernoulli's theorem (Eq.(21)) together with the continuity equation. Analogously to [31], the dimensionless water mass content in the tank is computed as

$$
M^{*}(t)=\frac{1}{H}\left(\sqrt{H}-\frac{A_{o}}{2} \sqrt{\frac{2 g}{A_{f s}^{2}-A_{o}^{2}} t}\right)^{2}
$$

where $A_{f s}=1 m^{2}$ is the area of the free-surface, and $A_{o}=C_{d} \pi D^{2} / 4$ is the effective area of the orifice. For sharp orifices, the discharge coefficient $C_{d}$ is generally considered between 0.60 and 0.65 . In this study $C_{d}=0.61$ has been considered.

In Figure 22 the dimensionless mass of the fluid remaining in the tank is compared to the analytical one obtained with Eq.(22).

The graphs show very good agreement between the numerical and the analytical solutions. This proves the capability of the PFEM for predicting the effective area of leakage in tanks discharge problems.

The convergence of the PFEM-DEM scheme has been also verified. For this purpose, four additional meshes have been analyzed for $\rho^{*}=1.0$ and $\rho^{*}=1.5$. These tessellations have mean mesh sizes $h=0.02 \mathrm{~m}, h=0.0275 \mathrm{~m}, h=$ $0.035 \mathrm{~m}$ and $h=0.045 \mathrm{~m}$, which correspond to $1249676,480698,233411$ and 109483 tetrahedral elements, respectively. Figure 23b shows the dimensionless velocity of the sample particle obtained with the five meshes. A logarithmic scale is used for the $y$-axis. The plot shows a clear convergent behavior of the PFEM-DEM scheme in both cases. The logarithmic scale also helps to 
(a)
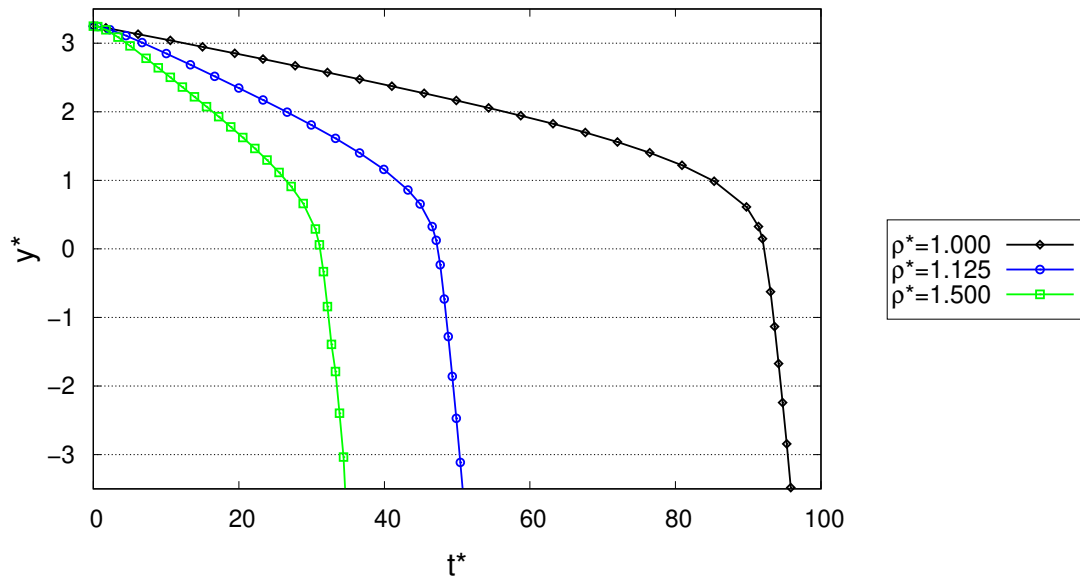

(b)

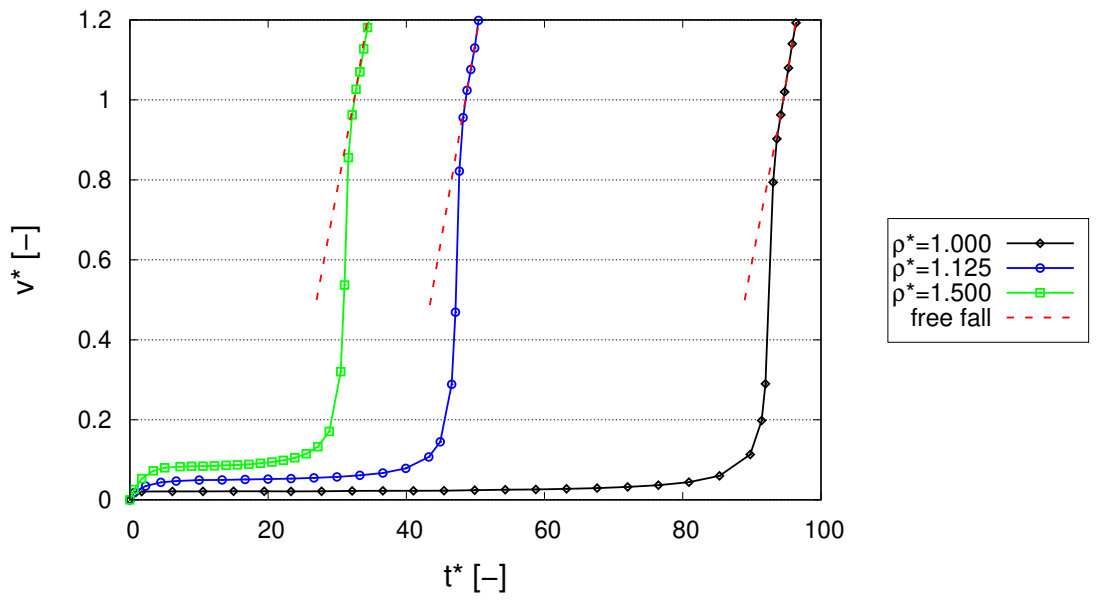

Fig. 21: Tank discharge. Dimensionless height and velocity of the sample particle for three values of density.

visualize better the different phases of the particle sedimentation described in the previous paragraphs.

\section{Conclusions}

This work has presented a fully Lagrangian formulation for the simulation of particle-laden flows with free surface. The focus has been placed on particle 


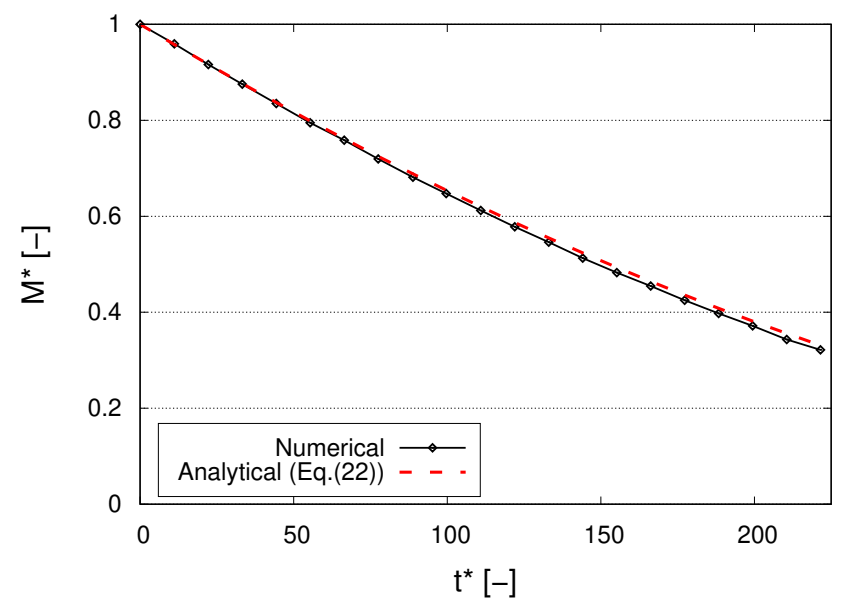

Fig. 22: Tank discharge. Time evolution of the dimensionless mass of the fluid remaining in the tank. Numerical and analytical results.

suspensions so small and disperse that their effect over the fluid motion can be considered as negligible (one-way coupling).

An implicit PFEM approach is used for modeling the free-surface flow, while the solid particles are modeled as spheres and computed explicitly with the DEM. A sub-stepping algorithm is used for reducing the computational time of the analyses.

In contrast to previous works where the spherical particles were overlapped to the PFEM nodes, here the solid spheres are free to move independently from the fluid mesh, thus no restrictions are imposed on the PFEM remeshing.

Four numerical tests have been studied in detail to highlight the capabilities and the accuracy of the coupled PFEM-DEM procedure.

In the first test, it has been proved that the sedimentation of a solid sphere in the Stoke's regime can be accurately reproduced with the method.

In the second test, the effect of the PFEM remeshing on the solution accuracy has been addressed. It has been shown that the changes of the internal connectivity of the PFEM mesh induce a certain perturbation of the PFEMDEM solution, however, the small shock produced by the remesh is quickly absorbed after a transient phase.

The third test, a water dam-break problem with embedded particles, has been used to validate the PFEM fluid solver for a complex 3D and highly unsteady free-surface flow. Good agreement with the experimental and published numerical results has been found. The DEM solution has been provided to allow future comparisons with other CFD-DEM solvers.

With the last test, it has been proved that the PFEM solver can model with very good accuracy the dynamics of a tank discharge, predicting with high fidelity the outlet velocity, the formation of the vena contracta and the fluid mass leakage time evolution. The motion of clouds of particles of different 
(a) $\rho^{*}=1.0$

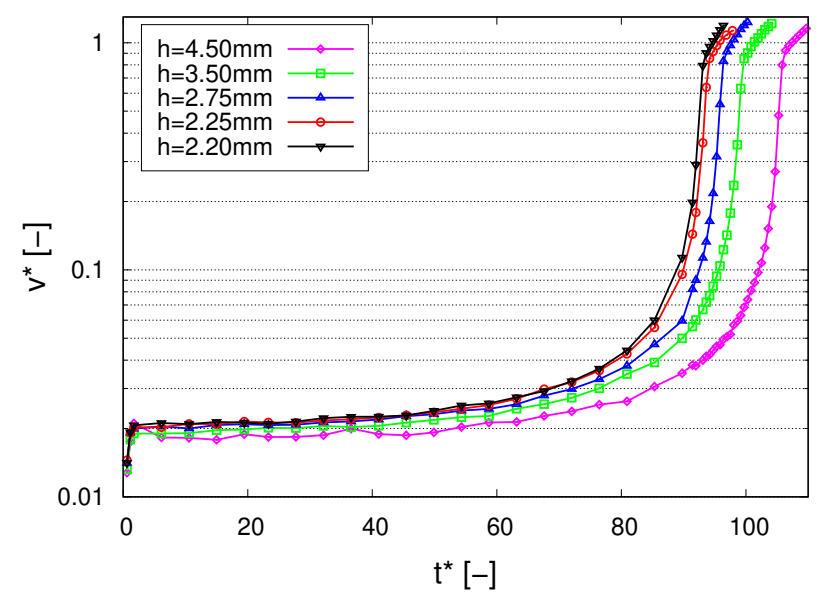

(b) $\rho^{*}=1.5$

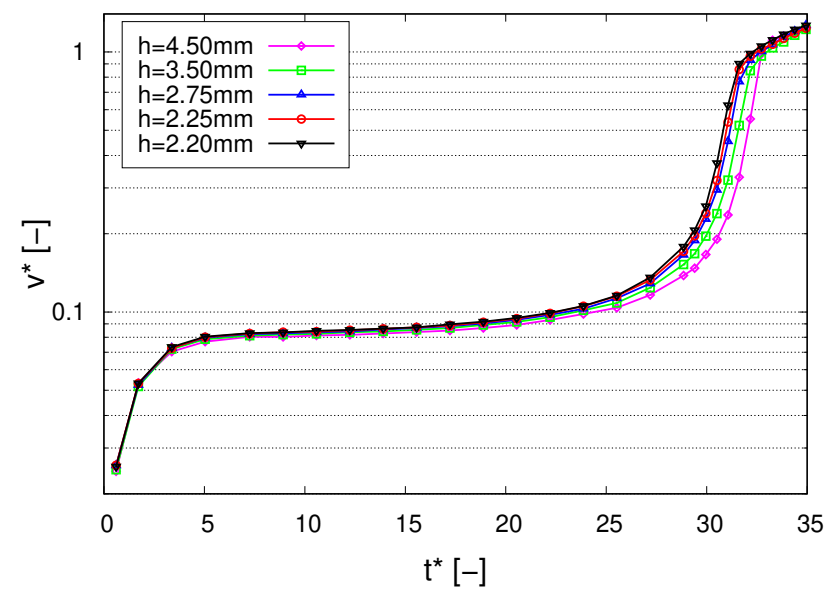

Fig. 23: Tank discharge. Dimensionless velocity of the sample particle for different mesh sizes and densities.

densities embedded in the fluid has been studied and compared. Moreover, it has been shown that the particles with the same density as the fluid can be used as tracers of the PFEM fluid solution. Finally, the convergent behavior of the PFEM-DEM scheme has been shown.

Future developments on the PFEM-DEM model here presented foresee to extend the numerical formulation to fully-coupled CFD-DEM analyses. This will allow us to solve problems where the effect of the motion of the particles on the fluid solution cannot be neglected. 


\section{A Appendix}

All vectors and matrices introduced in Section 3 are listed below. The variables used in the following refer to the fluid domain, hence the subindex $f$ is here omitted $\left(e . g . \rho=\rho_{f}\right)$.

The fully-discretized form of the linear momentum equations (Eq.(1)) reads

$$
K \Delta \bar{u}=R
$$

where $\boldsymbol{K}$ is computed as the sum of stiffness and mass matrices as

$$
\boldsymbol{K}=\boldsymbol{K}^{m}+\boldsymbol{K}^{\rho}
$$

with

$$
\begin{gathered}
\boldsymbol{K}_{I J}^{\rho}=\boldsymbol{I} \int_{\Omega} N_{I} \frac{2 \rho}{\Delta t} N_{J} d \Omega \\
\boldsymbol{K}_{I J}^{m}=\int_{\Omega} \boldsymbol{B}_{I}^{T} \boldsymbol{c} \boldsymbol{B}_{J} d \Omega
\end{gathered}
$$

and $\boldsymbol{R}$ is the residual of the discretized linear momentum equations computed at each iteration $i$ as

$$
R_{I i}^{i}=\int_{\Omega} N_{I} \rho N_{J} d \Omega \overline{\dot{u}}_{J i}^{i}+\int_{\Omega} \frac{\partial N_{I}}{\partial x_{j}} \sigma_{i j}^{i} d \Omega-\int_{\Omega} N_{I} \rho g_{i} d \Omega
$$

where $N_{I}$ are the linear shape functions and, for a $3 \mathrm{D}$ problem, $\boldsymbol{B}$ and $\boldsymbol{c}$ are defined as follows

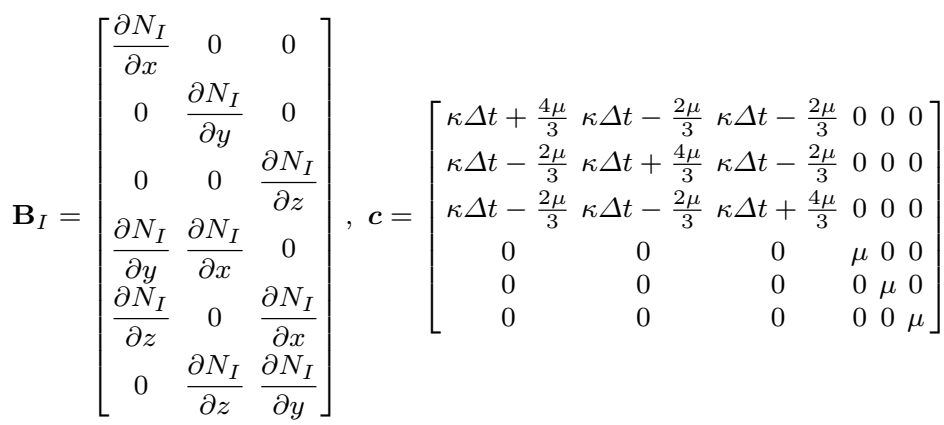

The fully-discretized form of the continuity equation (Eq.(4)) reads

$$
\boldsymbol{H} \overline{\boldsymbol{p}}=\boldsymbol{F}_{p}
$$

where

$$
\begin{gathered}
\boldsymbol{H}=\left(\frac{1}{\Delta t} \mathbf{M}_{1}+\frac{1}{\Delta t^{2}} \mathbf{M}_{2}+\mathbf{L}+\mathbf{M}_{b}\right) \\
\boldsymbol{F}_{p}=\frac{\mathbf{M}_{1}}{\Delta t} \overline{\boldsymbol{p}}^{n}+\frac{\mathbf{M}_{2}}{\Delta t^{2}}\left(\overline{\boldsymbol{p}}^{n}+\overline{\dot{p}}^{n} \Delta t\right)+\boldsymbol{f}_{p}-\mathbf{Q}^{T} \overline{\boldsymbol{v}}^{n+1}
\end{gathered}
$$

with

$$
\begin{gathered}
M_{1_{I J}}=\int_{\Omega} \frac{1}{\kappa} N_{I} N_{J} d \Omega \\
M_{2_{I J}}=\int_{\Omega} \tau \frac{\rho}{\kappa} N_{I} N_{J} d \Omega \\
L_{I J}=\int_{\Omega} \tau\left(\nabla^{T} N_{I}\right) \nabla N_{J} d \Omega
\end{gathered}
$$




$$
M_{b_{I J}}=\int_{\Gamma_{t}} \frac{2 \tau}{h_{n}} N_{I} N_{J} d \Gamma
$$

where $\Gamma_{t}$ is the free-surface contour and the stabilization parameter $\tau$ is defined as

$$
\tau=\left(\frac{8 \mu}{h^{2}}+\frac{2 \rho}{\delta}\right)^{-1}
$$

where $h$ and $\delta$ are characteristic distances in space and time [17].

Finally,

$$
\begin{gathered}
f_{p_{I}}=\int_{\Gamma_{t}} \tau N_{I}\left[\rho \frac{D u_{n}}{D t}-\frac{2}{h_{n}}\left(2 \mu d_{n}-\hat{t}\right)\right] d \Gamma-\int_{\Omega} \tau \nabla^{T} N_{I} \rho \boldsymbol{g} d \Omega \\
\mathbf{Q}_{I J}=\int_{\Omega} \mathbf{B}_{I}^{T} \mathbf{m} N_{J} d \Omega
\end{gathered}
$$

where in $3 \mathrm{D} \mathbf{m}=[1,1,1,0,0,0]^{T}$

Details on the derivation of above matrices and vectors can be found in [17].

\section{Acknowledgments}

The first author, beneficiary of an AXA Research Fund post-doctoral grant (grant number 2017-AXA-PDOC1-099), acknowledges the company for its economic support. 


\section{References}

1. S.R. Idelsohn and E. Oñate and F. Del Pin, The particle finite element method: a powerful tool to solve incompressible flows with free-surfaces and breaking waves, International Journal for Numerical Methods in Engineering, 61, 964-989 (2004)

2. E. Oñate and S.R. Idelsohn and F. Del Pin and R. Aubry, The particle finite element method. An overview, International Journal for Computational Methods, 1, 267-307 (2004)

3. A. Larese and R. Rossi and E. Oñate and S.R. Idelsohn, Validation of the particle finite element method (PFEM) for simulation of free surface flows, International Journal for Computer-Aided Engineering and Software, 25, 385-425 (2008)

4. S.R. Idelsohn and N. Calvo and E. Oñate, Polyhedrization of an arbitrary point set, Computer Methods in Applied Mechanics and Engineering, 92 (22-24), 2649-2668 (2003)

5. S. Meduri and M. Cremonesi and U. Perego and O. Bettinotti and A. Kurkchubasche and V.M. Oancea, A partitioned fully explicit Lagrangian finite element method for highly nonlinear fluid-structure interaction problems, International Journal for Numerical Methods in Engineering, 113, 43-64 (2018)

6. E. Oñate and M.A. Celigueta and S.R. Idelsohn, Modeling bed erosion in free surface flows by the Particle Finite Element Method, Acta Geotechnia, 1 (4), 237-252 (2006)

7. L. Monforte and M. Arroyo and J.M. Carbonell and A. Gens, Coupled effective stress analysis of insertion problems in geotechnics with the Particle Finite Element Method, Computers and Geotechnics, 101, 114-129 (2018)

8. X. Zhang and K. Krabbenhoft and D.M. Pedroso and A.V. Lyamin and D. Sheng and M. Vicente da Silva and D. Wang, Particle Finite element analysis of large deformation and granular flow problems, Computer and Geotechnics, 54, 133-142 (2013)

9. A. Franci and M. Cremonesi, 3D regularized $\mu(\mathrm{I})$-rheology for granular flows simulation, Journal of Computational Physics, 378, 257-277 (2019)

10. M. Zhu and M. H. Scott, Modeling fluid-structure interaction by the particle finite element method in OpenSees, Computers and Structures, 132, 12-21 (2014)

11. S.R. Idelsohn and J. Marti and A. Limache and E. Oñate, Unified Lagrangian Formulation For Elastic Solids And Incompressible Fluids: Applications to Fluid-Structure Interaction Problems Via The PFEM, Computer Methods In Applied Mechanics And Engineering, 197, 1762-1776 (2008)

12. P. Ryzhakov and E. Oñate and S.R. Idelsohn, Improving Mass Conservation in Simulation of Incompressible flows, International Journal of Numerical Methods in Engineering, 90, 1435-1451 (2012)

13. A. Franci and E. Oñate and J.M. Carbonell, On the effect of the bulk tangent matrix in partitioned solution schemes for nearly incompressible fluids, International Journal for Numerical Methods in Engineering, 102 (3-4), 257-277 (2015)

14. H. Edelsbrunner and E.P. Mucke, Three dimensional alpha shapes, ACM Trans Graphics, 13, 43-72, (1999)

15. H. Edelsbrunner and T.S. Tan, An upper bound for conforming Delaunay triangulations, Discrete and Computational Geometry, 10 (2), 197-213 (1993)

16. F. Brezzi, On The Existence, Uniqueness And Approximation Of Saddle-Point Problems Arising From Lagrange Multipliers, Revue française d'automatique, informatique, recherche opérationnelle. Série rouge. Analyse numérique, 8 (R-2), 129-151 (1974)

17. E. Oñate and A. Franci and J.M. Carbonell, Lagrangian formulation for finite element analysis of quasi-incompressible fluids with reduced mass losses, International Journal for Numerical Methods in Fluids, 74 (10), 699-731 (2014)

18. E. Oñate, Derivation of stabilized equations for advective-diffusive transport and fluid flow problems, Computer methods in applied mechanics and engineering, 151, 233-267 (1998)

19. I. De Pouplana and E. Oñate, A FIC-based stabilized mixed finite element method with equal order interpolation for solid-pore fluid interaction problems, International Journal for Numerical and Analytical Methods in Geomechanics, 41, 110-134 (2017)

20. A. Franci and M. Cremonesi, On the effect of standard PFEM remeshing on volume conservation in free-surface fluid flow problems, Computational Particle Mechanics, 4 (3), 331-343 (2017) 
21. M. Robinson and M. Ramaioli and S. Luding, Fluid-particle flow simulations using twoway-coupled mesoscale SPH-DEM and validation, International Journal of Multiphase Flow, 59, 121-134 (2014)

22. W.T. Ding and W.J. Xu, Study on the multiphase fluid-solid interaction in granular materials based on an LBM-DEM coupled method, Powder Technology, 335, 301-314 (2018)

23. A.M. Aly and M. Asai, Water entry of decelerating spheres simulations using improved ISPH method, Journal of Hydrodynamics, 30(6), 1120-1133 (2018)

24. J.C. Martin and W.J. Moyce, An experimental study of the collapse of liquid columns on a rigid horizontal plane 4, Philosophical Transactions of the Royal Society of London. Series A, 244, 312-324 (1952)

25. S. Koshizuka and Y. Oka, Moving-particle semi-implicit method for fragmentation of incompressible fluid, Philosophical Nuclear science and engineering, 123 (3), 421-434 (1996)

26. C. Hu and M. Sueyoshi, Numerical simulation and experiment on dam break problem, Journal of Marine Science and Application,9 (2), 109-114 (2010)

27. C.W. Hirt,B.D. Nichols, Volume of fluid (VOF) method for the dynamics of free boundaries. Journal of Computational Physics, 39, 201-225 (1981)

28. S. Koshizuka and Y. Oka, Moving particle semi-implicit method: fully Lagrangian analysis of incompressible flows, Proceedings of the European Congress on Computational Methods in Applied Sciences and Engineering (ECCOMAS), Barcelona (Spain) (2000)

29. M.L. Cerquaglia and G. Deliége and R. Boman and V. Terrapon and J.P. Ponthot, Free-slip boundary conditions for simulating free-surface incompressible flows through the particle finite element method, International Journal for Numerical Methods in Engineering, 110 (10), 921-946 (2016)

30. S. Meduri and M. Cremonesi and U. Perego, An efficient runtime mesh smoothing technique for 3D explicit Lagrangian free-surface fluid flow simulations, International Journal for Numerical Methods in Engineering, 113, 43-64 (2018)

31. A.M. Cervantes and S. Hidalgo and F. Pacheco, The simultaneous discharge of liquid and grains from a silo, Physics of Fluids, 30, 043302 (2018)

32. E. Loth and A. J. Dorgan, An equation of motion for particles of finite Reynolds number and size, Environmental Fluid Mechanics 9.2, 187-206 (2009)

33. E. Loth, Drag of non-spherical solid particles of regular and irregular shape, Powder Technology 182.3, 342-353 (2008)

34. N. G. Deen and E. A. J. F. Peters and J. T. Padding and J. A. M. Kuipers, Review of direct numerical simulation of fluid-particle mass, momentum and heat transfer in dense gas-solid flows, Chemical Engineering Science, 116, : 710-724 (2014)

35. T. B. Anderson and R. Jackson, Fluid mechanical description of fluidized beds. Equations of motion, Industrial \& Engineering Chemistry Fundamentals, 6.4, 527-539 (1967)

36. D. A. Drew and S. L. Passman, Theory of multicomponent fluids, Springer, New York (1999)

37. G. Casas, Numerical analysis of particle-laden flows with the finite element method, Doctoral Thesis, Polytechnic University of Catalonia, Barcelona (2018)

38. G. Casas, D. Mukherjee, M. A. Celigueta, T. I. Zohdi, and E. Onate. A modular, partitioned, discrete element framework for industrial grain distribution systems with rotating machinery. Computational particle mechanics, 4(2), 181-198 (2017)

39. E. Oñate, M.A. Celigueta, S. Latorre, G. Casas, R. Rossi, and J. Rojek. Lagrangian analysis of multiscale particulate flows with the particle finite element method. Computational Particle Mechanics, 1(1), 85-102 (2014)

40. M. A. Celigueta and K. M. Deshpande and S. Latorre and E. Oñate, A FEM-DEM technique for studying the motion of particles in non-Newtonian fluids. Application to the transport of drill cuttings in wellbores, Computational particle mechanics 3.2, 263-276 (2016)

41. C. Thornton, S. J. Cummins and P. W. Cleary, An investigation of the comparative behaviour of alternative contact force models during elastic collisions. Powder Technology, 210(3), 189-197 (2011)

42. J. R. Williams, E. Perkins and B. Cook, A contact algorithm for partitioning N arbitrary sized objects, Engineering Computations, 21 (2/3/4), 235-248 (2004)

43. J. Bec, Fractal clustering of inertial particles in random flows, Physics of fluids, 15.11, L81-L84 (2003) 
44. S. Radl and S. Sundaresan, A drag model for filtered Euler-Lagrange simulations of clustered gas-particle suspensions, Chemical engineering science, 117, 416-425 (2014)

45. H. R. Norouzi and R. Zarghami and R. Sotudeh-Gharebagh and N. Mostoufi, Coupled CFD-DEM modeling: formulation, implementation and application to multiphase flows, John Wiley \& Sons (2016)

46. P. J. Ireland and O. Desjardins, Improving particle drag predictions in Euler-Lagrange simulations with two-way coupling, Journal of Computational Physics 338, 405-430 (2017)

47. S. Radl and M. Girardi and S. Sundaresan, Effective Drag Law for Parcel-Based Approaches-What Can We Learn from CFD-DEM?, ECCOMAS 2012 (2012)

48. W. D. Fullmer and G. Liu and X. Yin and C. M. Hrenya, Clustering instabilities in sedimenting fluid-solid systems: critical assessment of kinetic-theory-based predictions using direct numerical simulation data, Journal of Fluid Mechanics 823, 433-469 (2017)

49. T. Mckeen and T. Pugsley, Simulation and experimental validation of a freely bubbling bed of FCC catalyst, Powder Technology 129.1-3, 139-152 (2003)

50. Z. Y. Zhou and S. B. Kuang and K. W. Chu and A. B. Yu, Discrete particle simulation of particle-fluid flow: model formulations and their applicability, Journal of Fluid Mechanics $661,482-510(2010)$

51. E. Epelle and D. I. Gerogiorgis, CFD modelling and simulation of drill cuttings transport efficiency in annular bends: Effect of particle sphericity, Journal of Petroleum Science and Engineering (2018)

52. N. Gui and F. JianRen and C. Kefa, Effect of particle-particle collision in decaying homogeneous and isotropic turbulence, Physical Review E, 78.4, 046307 (2008)

53. A. G. Kidanemariam and C. Chan-Braun and T. Doychev and M. Uhlmann, Direct numerical simulation of horizontal open channel flow with finite-size, heavy particles at low solid volume fraction, New Journal of Physics 15.2, 025031 (2013)

54. F. Alobaid, A particle-grid method for Euler-Lagrange approach, Powder Technology 286, 342-360 (2015)

55. S. Lain and M. Sommerfeld, Turbulence modulation in dispersed two-phase flow laden with solids from a Lagrangian perspective, International Journal of Heat and Fluid Flow 24.4, 616-625 (2003)

56. O. Vermorel and B. Bedat and O. Simonin and T. Poinsot, Numerical study and modelling of turbulence modulation in a particle laden slab flow, Journal of Turbulence 4.25, $1-39(2003)$

57. D. M. Snider, An incompressible three-dimensional multiphase particle-in-cell model for dense particle flows, Journal of Computational Physics 170.2, 523-549 (2001)

58. L. Wakaba and S. Balachandar, On the added mass force at finite Reynolds and acceleration numbers, Theoretical and Computational fluid dynamics, 21.2, 147-153 (2007)

59. A. A. Kendoush, The virtual mass of an oblate-ellipsoidal bubble, Physics Letters A 366.3, 253-255 (2007)

60. R. Beetstra and M. A. van der Hoef and J. A. M. Kuipers, Drag force of intermediate Reynolds number flow past mono-and bidisperse arrays of spheres, AIChE journal 53.2, 489-501 (2007)

61. X. Sun and M. Sakai and Y. Yamada, Three-dimensional simulation of a solid-liquid flow by the DEM-SPH method, Journal of Computational Physics, 248, 147-176 (2013)

62. Y. He and A.E. Bayly and A. Hassanpour and F. Muller and K. Wu and D. Yang, A GPU-based coupled SPH-DEM method for particle-fluid flow with free surfaces, Powder Technology, 338, 548-562 (2018)

63. L. Shiller and A. Naumann, A Drag Coefficient Correlation. Zeitschrift des Vereins Deutscher Ingenieure, 77, 318-320 (1935)

64. E. Hairer and C. Lubich and G. Wanner. Geometric numerical integration illustrated by the Störmer-Verlet method. Acta numerica, 12, 399-450 (2003)

65. F.H. Harlow, A Machine Calculation Method for Hydrodynamic Problems, Los Alamos Scientific Laboratory report LAMS-1956 (1955)

66. S. Elghobashi, On predicting particle-laden turbulent flows, Applied scientific research 52.4, 309-329 (1994)

67. T. Kawaguchi and T. Tanaka and Y. Tsuji, Numerical simulation of two-dimensional fluidized beds using the discrete element method (comparison between the two-and threedimensional models), Powder technology 96.2, 129-138 (1998) 